\title{
A data-adaptive robust unit commitment model considering high penetration of wind power generation and its enhanced uncertainty set
}

\author{
Lin, Zhenjia; Chen, Haoyong; Wu, Qiuwei; Huang, Jianping; Li, Mengshi; Ji, Tianyao
}

Published in:

International Journal of Electrical Power \& Energy Systems

Link to article, DOI:

10.1016/j.ijepes.2021.106797

Publication date:

2021

Document Version

Peer reviewed version

Link back to DTU Orbit

Citation $(A P A)$ :

Lin, Z., Chen, H., Wu, Q., Huang, J., Li, M., \& Ji, T. (2021). A data-adaptive robust unit commitment model considering high penetration of wind power generation and its enhanced uncertainty set. International Journal of Electrical Power \& Energy Systems, 129, [106797]. https://doi.org/10.1016/j.ijepes.2021.106797

\section{General rights}

Copyright and moral rights for the publications made accessible in the public portal are retained by the authors and/or other copyright owners and it is a condition of accessing publications that users recognise and abide by the legal requirements associated with these rights.

- Users may download and print one copy of any publication from the public portal for the purpose of private study or research.

- You may not further distribute the material or use it for any profit-making activity or commercial gain

- You may freely distribute the URL identifying the publication in the public portal 


\title{
A data-adaptive robust unit commitment model considering high penetration of wind power generation and its enhanced uncertainty set
}

\author{
Zhenjia Lin ${ }^{\mathrm{a}}$, Haoyong Chen ${ }^{\mathrm{a}, *}$, Qiuwei $\mathrm{Wu}^{\mathrm{b}}$, Jianping Huanga ${ }^{\mathrm{a}}$, Mengshi $\mathrm{Li}^{\mathrm{a}}$, Tianyao $\mathrm{Ji}^{\mathrm{a}}$ \\ ${ }^{a}$ School of Electric Power Engineering, South China University of Technology, Guangzhou 510000, China \\ ${ }^{b}$ Center for Electric Power and Energy, Department of Electrical Engineering, Technical University of \\ Denmark, Kgs. Lyngby DK 2800, Denmark
}

\begin{abstract}
Wind power generation is increasingly penetrating into the power grid, which brings great challenges to the dispatch of power systems. With the popularization of data mining technology, further exploration of the random characteristics of wind power based on the available wind power data can significantly improve the applicability of scheduling decisions. In this paper, a novel data-adaptive robust unit commitment model under high penetration of wind power is proposed, which derives a robust dispatch solution with minimal generation cost while hedging against the worst case in the uncertainty set. Firstly, copula theory is carried out to formulate a joint probabilistic distribution function and capture the correlation of power outputs among multiple wind farms. A large number of wind power scenarios are then generated and the imprecise Dirichlet model (IDM) is applied to derive the boundaries of wind power generation, which helps to construct a more practical polyhedron uncertainty set. Moreover, due to the correlation of adjacent wind farms, the auxiliary variables which determine the fluctuation of wind power have a synchronous trend. Here, the synchronous characteristic is introduced to the enhanced polyhedron uncertainty set by means of the synchronous volatility of the auxiliary variables in adjacent wind farms. Experimental studies are conducted out on a modified IEEE-118 bus system and the obtained scheduling solution is turned out to be superior under wind power uncertainties, which verifies the effectiveness of the proposed data-adaptive robust unit commitment model.
\end{abstract}

Keywords: High wind power penetration, robust scheduling, the correlation of wind power, the enhanced uncertainty set.

${ }^{*}$ Corresponding author: Pro. Haoyong Chen. This work is financially supported by the National Natural Science Foundation of China (51937005), the National Key Research and Development Program of China (2016YFB0900100) and the Oversea Study Program of Guangzhou Elite Project (GEP).

Email address: eehychen@scut.edu.cn (Haoyong Chen) 


\section{Introduction}

Due to the global energy crisis and the natural environment problem caused by the greenhouse effect, the large scale utilization of renewable energy resources has been an irreversible trend in energy strategy [1]. On one hand, with the development of electric power technology, the penetration of renewable energy in the power grid is constantly increasing, which is conductive to reducing the dependence on traditional fossil energy $[2,3]$. On the other hand, the large scale integration of stochastic renewable energy into the power grid has caused great uncertainties to the power systems, which brings great challenges to the dispatch of power systems $[4,5]$.

Take the wind power as an example, system operators have to deploy excessive system reserve capacity to adapt to the random fluctuations of wind power, which often results in high generation costs. At the same time, in order to make room for the spinning reserve capacity, the generator units have to make compromises and cannot operate effectively at the optimal working point. In this regard, to determine an optimal scheduling scheme, the random characteristics of wind power should be taken into account. There are two main approaches used to tackle the uncertainties, namely stochastic optimization (SO) and robust optimization (RO). The former generates a series of wind power scenarios to represent the uncertainties of wind power based on the Monte Carlo simulations (MCS) [6], and seeks a dispatch scheme with optimal expected cost efficiency through the probabilistic information on random variables. However, it is difficult to estimate the exact probabilistic distribution of random variables, and the scenario-based method converts the stochastic optimization problem into a number of deterministic ones, which turns out to be intractable as the sample size becomes very large $[7,8]$.

Alternatively, the worst-case based robust optimization derives an optimal solution that remains feasible for possible realizations of the uncertain parameters in the uncertainty set. It requires less information for the random variables and has been successfully applied to optimal power flow (OPF), unit commitment (UC) and economic dispatch (ED) problems $[9,10]$. RO is controversial since it has to hedge against the worst case and the resulting solution always turns to be overconservative. To tackle the conservatism, Bertsimas and Sim in [11] derive a robust formulation for uncertain linear programming problems using budget parameter, which can be changed in cardinality by varying the confidence level to adjust conservativeness of the uncertainty set. Many subsequent researches have devoted to alleviate the over-conservativeness of the RO approach. Refs. [12, 13] introduce the risk penalty cost into the objective function under the min-max-min mathematical framework to determine the optimal uncertainty set. Ref. [14] proposes a novel uncertainty set in which the uncertainty budget can be determined by a probabilistic number in terms that it restricts the total deviation in a probabilistic way. Despite this, it is critical to determine an appropriate uncertainty set for uncertain wind power generation, which is of great significance to the tradeoff between the robustness and economy [15]. Especially in the case of considering the adjacent wind farms, that is, when the power output of a wind farm increases, the output of other wind farms in adjacent areas also increases with a great probability. However, as illustrated in $[16,17]$, traditional uncertainty set in $\mathrm{RO}$ rarely considers the correlation of 
multiple wind farms, in which the power outputs are generally assumed to be independent of each other. The approaches in [18] and [19] consider the correlation of multiple wind farms in the robust scheduling optimization model, in which the correlation information is incorporated into the uncertainty set in terms of the second order moment. However, the obscure model makes it difficult to obtain a global optimal solution for the non-convexity of the objective problem.

The investigation of statistical information from the available data can potentially help to build a data-adaptive uncertainty set and improve the quality of decision-making [20, 21]. Zhang et al. in [22] has successfully applied the imprecise Dirichlet model (IDM) [23] to derive the lower and upper boundaries of wind power generation with a given confidence level based on the available historical wind power data. However, it is difficult for the IDM to obtain the convergent confidence intervals when the sample size of historical data is insufficient, which would lead to the inferior boundaries of wind power generation. Fortunately, the deficiency of IDM can be made up by the introduction of copula function, which is the first attempt in this paper to improve the applicability of the IDM method. Copula theory has attracted great attentions in the modeling of multivariate distribution in recent years. As indicated in $[24,25]$, the copula functions have been widely used to capture the dependence among multiple wind farms and achieve good performance. In this paper, based on the available wind power data, the copula function is applied to build the joint probability distribution function and captures the correlation of wind power among multiple wind farms for each scheduling time period. Then, the joint distribution function can be further used to generate the uncertain wind power scenarios by the quasi-Monte Carlo simulations method [26]. It should be noted that the wind power scenarios generated by copula function can make up the deficiency of insufficient historical wind power samples and enable the IDM method to derive the convergent confidence intervals of wind power generation. As a result, by extracting the useful information from the available wind power scenarios, we can obtain the more reliable boundaries of wind power generation, which can then be incorporated into the uncertainty set and alleviate the conservativeness of RO.

Moreover, as illustrated in [27], the worst case of RO is generally subjected to the vertices of the polyhedral uncertainty set, which are the random combinations of extreme scenarios of different wind farms. Due to the inertia of meteorological systems, the volatility of adjacent wind farms in geographic areas tends to fluctuate in a similar trend. However, traditional construction of uncertainty set ignores the synchronous characteristic, and the obtained dispatch decision tends to be over-conservative since many unnecessary extreme scenarios are included into the uncertainty set. In this paper, to eliminate the unnecessary vertices, the synchronous characteristic is incorporated into the enhanced polyhedron uncertainty set by means of the synchronous volatility of the corresponding auxiliary variables in adjacent wind farms.

Therefore, based on the enhanced uncertainty set, the proposed data-adaptive robust unit commitment (DARUC) model is formulated as a two-stage problem, among which the first stage determines the day-ahead unit commitment status, while the second stage solves economic dispatch problems after the realization of uncertain wind power generation. The column-and-constraint generation $(\mathrm{C} \& \mathrm{CG})$ algorithm enjoys a fast preserved convergence 
and achieves an efficient solution for a mixed-integer nonlinear programming problem [28]. In this paper, to verify the effectiveness of the proposed DARUC model, simulations are conducted on a modified IEEE 118-bus system and C\&CG is utilized to derive an optimal scheduling scheme.

The major contributions of this paper are as follow:

- The copula theory is utilized in this paper to mine the characteristics of the historical wind power data, and wind power scenarios are generated according to the formulated joint distribution function of wind power among multiple wind farms, which exactly makes up the defect of the imprecise Dirichlet model (IDM) in obtaining the convergent confidence intervals when insufficient historical data is available.

- Rather than assuming that the lower and upper bounds of wind power generation are specific values, the proposed method determines the boundaries of wind power generation based on the available wind power scenarios. In this regard, the data-adaptive boundaries of wind power generation can reflect the available information more objectively, and ultimately lead to a more practical polyhedron uncertainty set.

- The characteristic that the volatility in adjacent wind farms has a synchronous trend is taken in consideration in this paper by means of the synchronous volatility of corresponding auxiliary variables in adjacent wind farms. The unnecessary extreme scenarios are eliminated in the enhanced polyhedron uncertainty set so as to alleviate the conservativeness of the robust unit commitment model.

The rest of the paper are organized as follows: Section 2 elaborates the data-adaptive wind power generation considering the correlation among multiple wind farms, Section 3 illustrates the enhanced uncertainty set that incorporates the synchronous volatility of adjacent wind farms. In Section 4, the data-adaptive robust unit commitment model is proposed and the experimental studies are presented in Section 5. Finally, The last Section draws the conclusion of this paper.

\section{The data-adaptive wind power generation considering the correlation among multiple wind farms}

\subsection{The correlation among multiple wind farms}

Renewable energy sources account a more significant penetration in energy systems, among which the wind power generation is widely used for its abundant resources and easy access. Unlike thermal generation, wind power is characterized by its random power output, which has caused great uncertainties to the power systems. Typically, the wind power with high permeability is dispersedly integrated into multiple wind farms in adjacent areas. However, it is generally assumed that the uncertain power generation of different wind farms are independent of each other and one uncertain parameter could not exert on other uncertainties. In fact, the correlation may arise in the uncertain power outputs of multiple wind farms, especially the tail characteristics of wind power [24].

Fig. 1 illustrates the comparisons of power outputs among multiple wind farms from actual data and those generated without considering the correlations. It can be easily figured out that the power outputs in wind farm \#1 and wind farm \#2 are closely related to each 

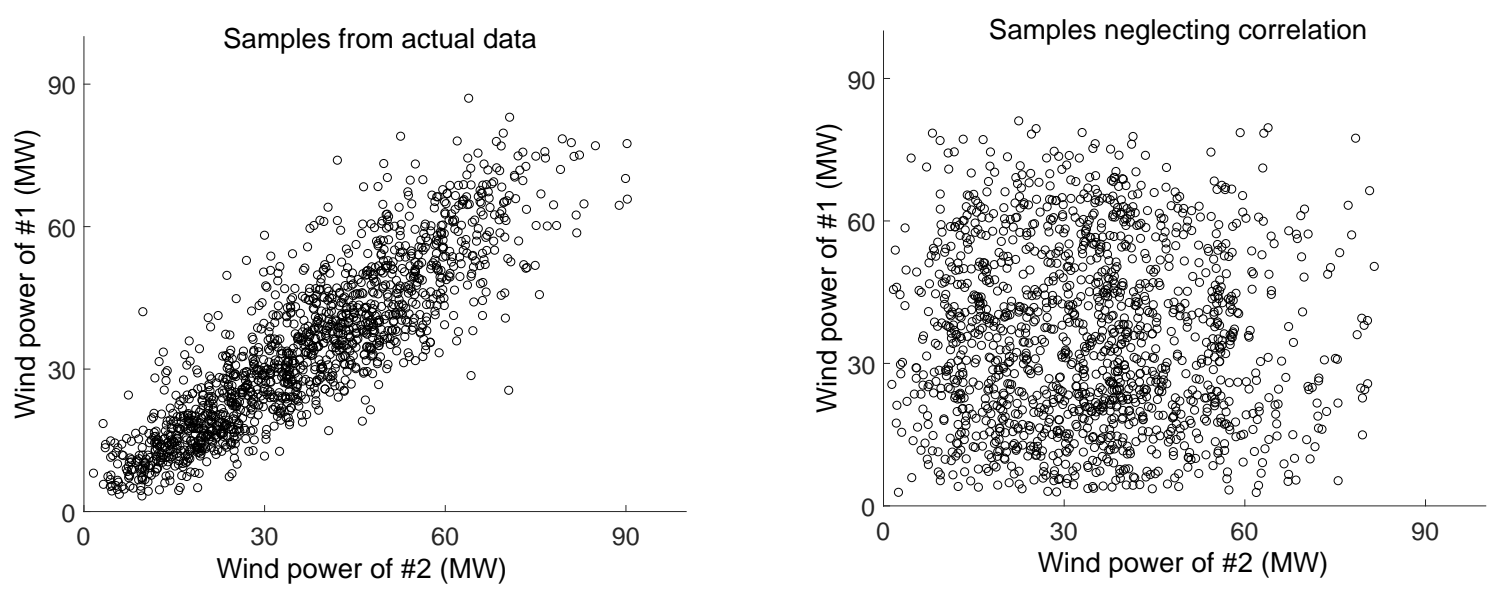

Figure 1: Comparisons of wind power samples among two wind farms

other. However, when we ignore the correlation of wind farms, just as shown in the right side of the figure, the power outputs of two wind farms are randomly distributed on the plane. When the power output is low or high in a wind farm, it has nothing to do with the power output of another wind farm. The correlation of wind farms affects the fluctuating range of wind power generation in the uncertainty set. Specifically, the inappropriate establishment of the uncertainty set would derive the over-conservative dispatch solutions, which may result in unnecessary wind curtailment and load shedding.

In this paper, copula function is applied to capture the correlation of wind power outputs among different wind farms for its great advantages in building the joint distribution function of multivariate. As illustrated in Sklar's theorem [29], for every $n$-dimensional distribution function $F(\cdot)$, with the univariate marginal cumulative distribution functions (CDFs) $F_{i}\left(x_{i}\right), i=1, \ldots, n$ representing the distribution function of power output of the $i^{\text {th }}$ wind farm, there exists a unique copula function $C(\cdot)$ if all marginal distributions are continuous, which is formulated as:

$$
F\left(x_{1}, x_{2}, \ldots, x_{n}\right)=C\left(F_{1}\left(x_{1}\right), F_{2}\left(x_{2}\right), \ldots, F_{n}\left(x_{n}\right)\right)
$$

where the multivariate function $F\left(x_{1}, x_{2}, \ldots, x_{n}\right)$ describing the joint distribution of wind power outputs that can be obtained by the copula function. However, there are a variety of copula functions in Elliptical copulas and Archimedean copulas, which are the two main clusters in the family tree of copula functions [30]. In this paper, t-copula is used to model the correlation of wind farms for its advantages in capturing the tail dependence feactures as well as flexibly extending to high dimensions, which is given as:

$$
C^{t}\left(u_{1}, u_{2}, \ldots, u_{n} ; \rho, k\right)=t_{\rho, k}\left(t_{k}^{-1}\left(u_{1}\right), t_{k}^{-1}\left(u_{2}\right), \ldots, t_{k}^{-1}\left(u_{n}\right)\right)
$$

where $u_{i}$ is a shorthand for $F_{i}\left(x_{i}\right), i=1, \ldots, n$ that can be obtained through the nonparametric kernel density estimation $[30], t_{\rho, k}(\cdot)$ is the multivariate t-distribution function while $t_{k}^{-1}(\cdot)$ denotes the inverse function of the univariate t-distribution. For each scheduling 
time interval, the correlation matrix $\rho$ and the freedom degree $k$ of the t-copula function are determined by the maximum likelihood estimation method [26]. Therefore, the obtained joint distribution functions of high-dimensional uncertainties can be applied to generate the wind power scenarios of multiple wind farms, in which the correlation of wind farms has been fully considered.

For the $t^{\text {th }}$ scheduling time period, the t-copula function $C^{t}\left(u_{1}, u_{2}, \ldots, u_{n} ; \rho_{t}, k_{t}\right)$ can be easily figured out based on the historical wind power data. Then, the joint distribution function can be further used to generate the uncertain wind power scenarios based on the quasi-Monte Carlo simulations method. Note that the sampling procedure of wind power scenarios has been illustrated in detail in [31]. Here, our problem turns to how to obtain the data-adaptive range of wind power generation through the generated wind power scenarios, which will be addressed in the following section.

\subsection{The data-adaptive wind power generation}

In this paper, we assume that the system operators are accessible to the forecasted value of wind power as well as the historical wind power data, while the forecast intervals of wind power are not necessarily provided with them. Existing literatures generally predetermine the lower and upper deviations of wind power generation to be $\pm 30 \%$ or so of the forecasted value when constructing the uncertainty set. However, the subjective boundaries of wind power generation will greatly affect the conservativeness of the optimization. In this paper, the copula theory is utilized to mine the historical wind power data and capture the correlation characteristic of multiple wind farms, so that the generated wind power scenarios are more in line with the actual situations. In this regard, if we further extract information from the generated wind power scenarios, we can obtain the more reliable boundaries of wind power generation.

Fortunately, Walley in [23] proposed the imprecise probability theory to quantify the uncertainty of random event by an interval-valued probability, which can then be estimated by the imprecise Dirichlet model (IDM). Consider a random variable with $K$ types of possible states and their corresponding density functions $P_{k}, k=1,2, \ldots K$, the set of posterior density functions with respect to the observations $n$ belongs to a Dirichlet distribution, which can be estimated according to the Bayesian rules [32],

$$
f\left(P_{k}\right)=\frac{\Gamma(s+n)}{\prod_{k=1}^{K} \Gamma\left(s \cdot r_{k}+n_{k}\right)} \prod_{k=1}^{K} P_{k}^{s \cdot r_{k}+n_{k}-1}, \forall r_{k} \in[0,1], \sum_{1}^{K} r_{k}=1
$$

where $\Gamma(\cdot)$ is the Gamma function, $s$ determines the convergence rate of the upper and lower probabilities as the statistical data accumulates, which is set to 1 in this paper, $n_{k}$ is the number of the $k^{\text {th }}$ sample that is observed and $n$ represents the number of total observations, $r_{k}$ is the $k^{\text {th }}$ weight factor, when it varies in the interval $[0,1]$, all possible density functions can be included to the above set. As referred to [33], the posterior density function can be further estimated by:

$$
P_{k}=\frac{n_{k}+s \cdot r_{k}}{n+s}
$$


However, it is difficult to determine a proper value for $r_{k}$, especially if there are insufficient available observations, and an unreasonable prior setting of $r_{k}$ will affect the accuracy of $P_{k}$. To avoid the determination of $r_{k}$, IDM is proposed to include all the possible probabilities corresponding to different priors of $r_{k}$. Accordingly, $P_{k}$ is described in the form of probability intervals, which can be estimated from the posterior density set as:

$$
\widetilde{P}_{k}=\left[\underline{P}_{k}, \bar{P}_{k}\right]=\left[\frac{n_{k}}{n+s}, \frac{n_{k}+s}{n+s}\right], k=1,2, \ldots K
$$

where the lower and upper bounds of the probability interval, $\underline{P}_{k}$ and $\bar{P}_{k}$, are evaluated with respect to the bounds of $r_{k}$. Here, the width of the probability interval depends on the sample size of the observations. Despite this, the probability interval with a quantitative confidence index may be preferred in some applications. In that case, the confidence interval $\left[a_{k}, b_{k}\right]$ of the cumulative probability with a given confidence level $\gamma$ can be estimated as:

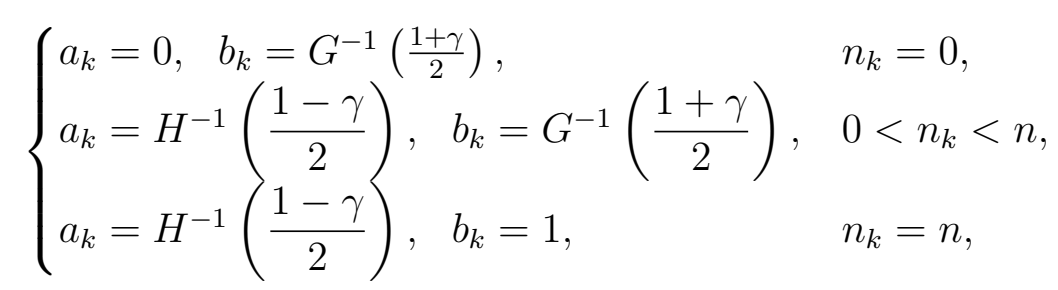

where $H$ and $G$ are the CDFs of Beta distribution functions $B\left(n_{k}, s+n-n_{k}\right)$ and $B(s+$ $\left.n_{k}, n-n_{k}\right)$, respectively.

It can be inferred that the probability intervals of $\left[a_{k}, b_{k}\right], k=1,2, \ldots K$ gradually narrow with the increase of the sample data, and finally a more accurate CDF can be derived, from which we can obtain the data-adaptive wind power generation. As shown in Fig. 2, when the data sample increases from 1000 to 2000, the confidence bounds are much closer to the true CDF, and the probability gap can be ignored when the data sample reaches a certain number. It should be noted that the wind power scenarios capturing the correlation of multiple wind farms can be generated as many as possible to acquire the convergent CDF in case that the actual data is usually insufficient. In this respect, the upper and lower bounds of wind power output with respect to the probability $(1+\gamma) / 2$ and $(1-\gamma) / 2$, can be obtained, as $\bar{p}_{w t}^{\mathrm{W}}$ and $p_{w t}^{\mathrm{W}}$ displayed in Fig. 2. Therefore, the data-adaptive wind power generation of different wind farms can be incorporated into the uncertainty set, which will become less conservative and result in more practical dispatch solutions when applied in the robust unit commitment model.

\section{The enhanced polyhedron uncertainty set}

The uncertainty set is the core concept of robust optimization, which aims to incorporate all possible information of uncertain parameters. However, traditional modeling of uncertainty set intends to cover all the possible scenarios that may occur under actual situations, which cannot reflect the exact distribution of uncertain variables [34]. Due to the correlation among uncertainties, many extreme scenarios in the original uncertainty set could be negligi- 


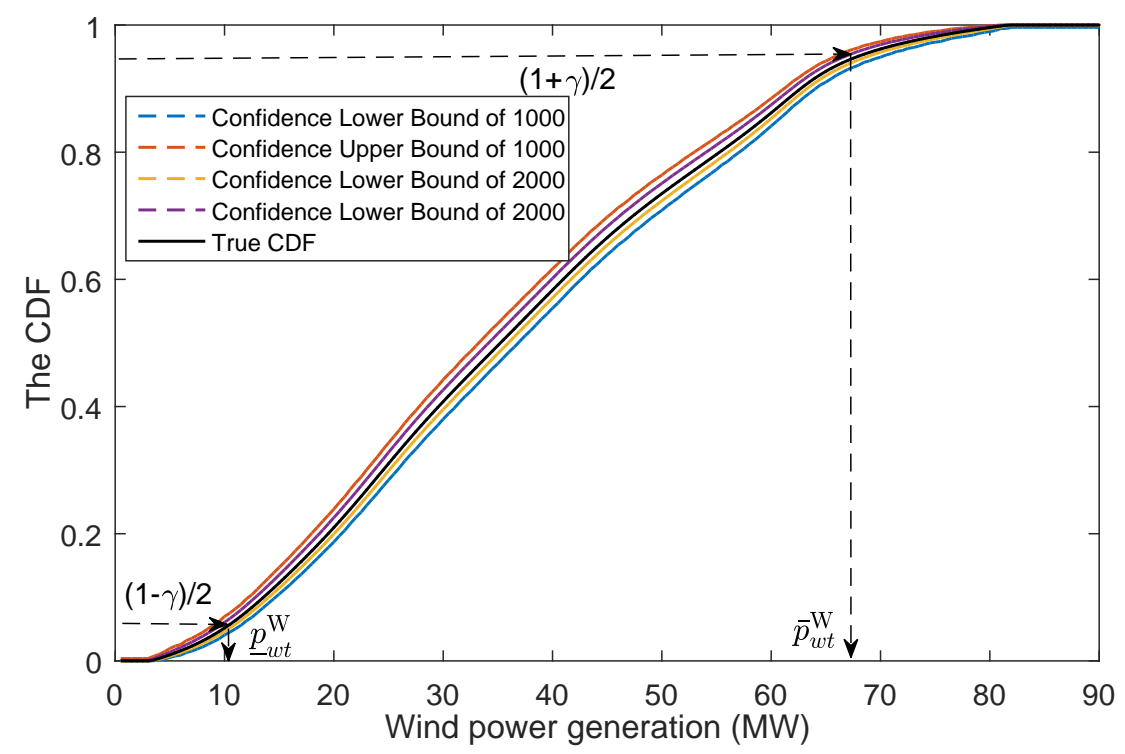

Figure 2: The wind power generation with a given confidence level $\gamma$

ble. These extreme points may formulate the worst case and lead to the inefficient operation of power systems, even the waste of computing resources. As a result, it is important to investigate strategies to remove the unnecessary extreme scenarios from the uncertainty set and reduce the conservativeness in robust optimization.

As the two wind farms indicated in Fig. 3, when all the error scenarios and extreme scenarios are included in the uncertainty set for the robust unit commitment model, the derived worst-case based robust scheduling solution can be immune to the random wind power. However, the power generations of two wind farms in adjacent area are actually closely related to each other and the fluctuation of two wind farms has a synchronous trend. In this regard, the worst case may be dominated by the extreme scenarios $\mathrm{S}_{2}$ and $\mathrm{S}_{4}$ when they are included into the uncertainty set, but they hardly take place in practice, which would drive the robust UC model to be over-conservative.

The wind power $p_{w t}^{\mathrm{W}}$ always fluctuates randomly between its upper bound $\bar{p}_{w t}^{\mathrm{W}}$ and lower bound $\underline{p}_{w t}^{\mathrm{W}}$, and the polyhedron uncertainty set of the uncertain wind power for the robust UC model is defined as follow:

$$
\begin{aligned}
\mathcal{U}^{w}=\{ & p_{w t}^{\mathrm{W}}, \forall w \in \mathcal{W}, t \in \mathcal{T} \mid \\
& p_{w t}^{\mathrm{W}}=\tilde{p}_{w t}^{\mathrm{W}}+\left(\bar{p}_{w t}^{\mathrm{W}}-\tilde{p}_{w t}^{\mathrm{W}}\right) \cdot z_{w t}^{+}-\left(\tilde{p}_{w t}^{\mathrm{W}}-\underline{p}_{w t}^{\mathrm{W}}\right) \cdot z_{w t}^{-}, \\
& \sum_{t \in \mathcal{T}}\left(z_{w t}^{+}+z_{w t}^{-}\right) \leq \Gamma_{w}, \sum_{w \in \mathcal{W}}\left(z_{w t}^{+}+z_{w t}^{-}\right) \leq \Pi_{t}, \\
& \left.\exists z_{w t}^{+}, z_{w t}^{-} \in[0,1], \forall w \in \mathcal{W}, t \in \mathcal{T}\right\}
\end{aligned}
$$

where $\tilde{p}_{w t}^{\mathrm{W}}$ represents the forecast value, $z_{w t}^{+}$and $z_{w t}^{-}$are auxiliary variables indicating the fluctuation of wind power, $\mathcal{W}$ represents the set of wind farms and $\mathcal{T}$ is the set of scheduling 


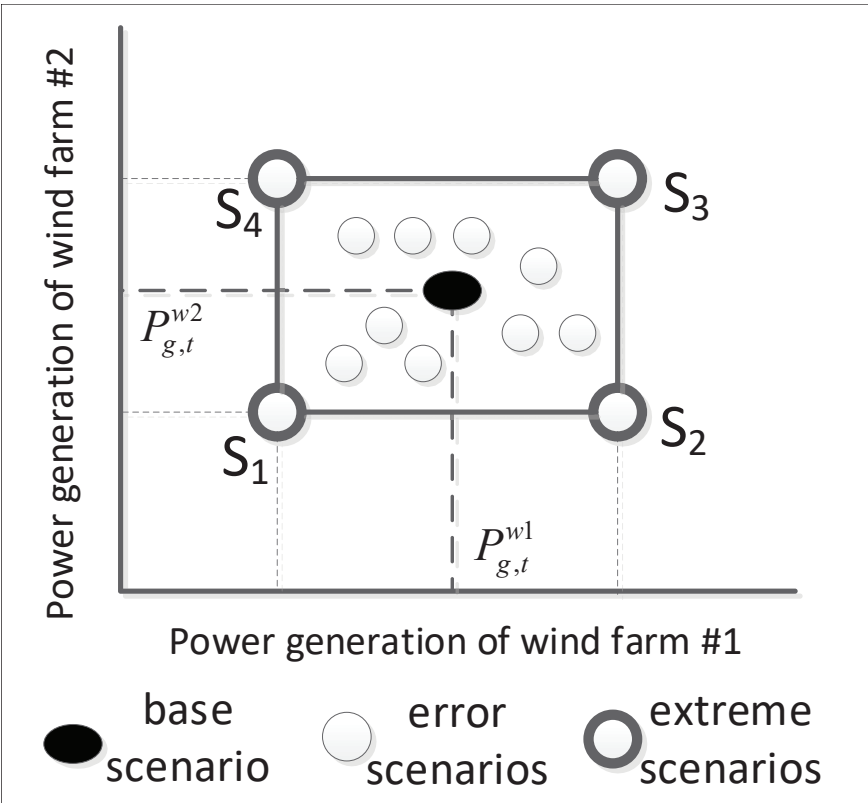

Figure 3: The possible wind power scenarios of two wind farms

periods, $\Gamma_{w}$ and $\Pi_{t}$ are the budgets for the temporal and spatial budget constraints, respectively, which provide a tradeoff between robustness and conservatism. Intuitively, the worst case is subjected to the vertices of the polyhedral uncertainty set, which are generally the random combinations of extreme scenarios of different wind farms.

However, as depicted in Fig. 4, there are two distant area $a$ and area $b$. For the adjacent wind farms $i, j, \ldots, k$ that cluster in area $a$ with abundant wind resources, they have positive/negative synchronous volatility in each time period due to the spatial correlation. In this paper, the synchronous characteristic is incorporated into the enhanced polyhedron uncertainty set by means of the synchronous volatility of auxiliary variables in adjacent wind farms. Here, we introduce the regional fluctuation variable $z_{a t}^{+}$to represent the positive volatility of area $a$ at time $t$, and the synchronous volatility is then converted to the distance between the regional fluctuation variable $z_{a t}^{+}$and the auxiliary variables $z_{w t}^{+}(w=i, j, \ldots, k)$ of each wind farm, which is elaborately formulated as:

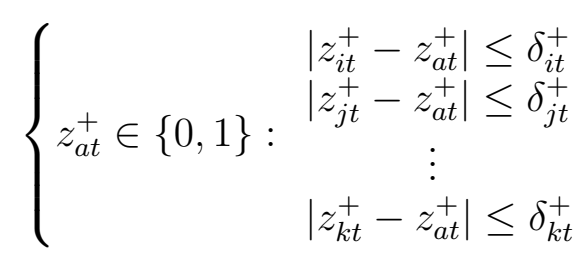

where $i, j, \ldots, k$ denote the adjacent wind farms in area $a, \delta_{w t}^{+}(w=i, j, \ldots, k)$ is a disturbance factor that determines the consistency of fluctuations of different wind farms. The smaller $\delta_{w t}^{+}$values, the closer the distance from $z_{w t}^{+}(w=i, j, \ldots, k)$ to $z_{a t}^{+}$, and the higher consistency of synchronous volatility of adjacent wind farms, which can exactly describe the 


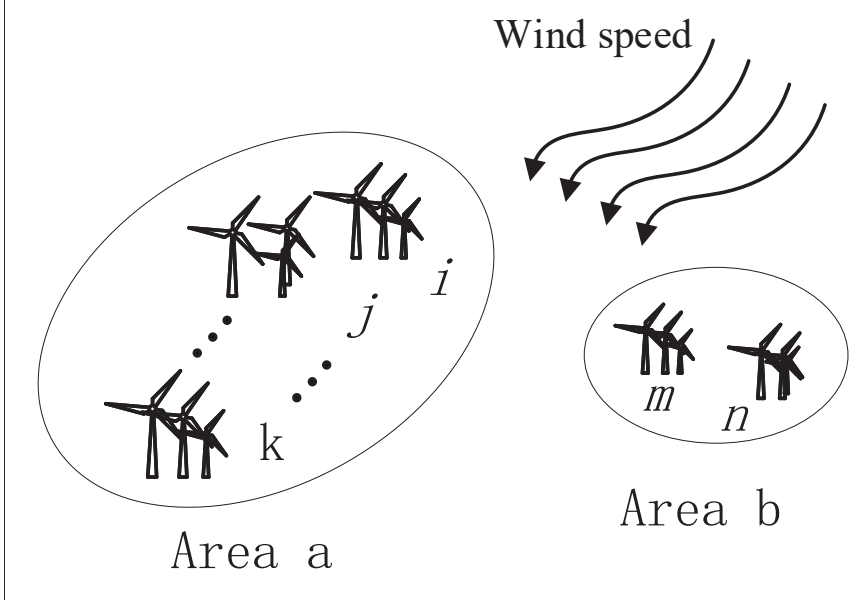

Figure 4: The synchronous volatility of adjacent wind farms

correlation of adjacent wind farms. Here, we assume that the topological distribution of wind farms is well known by the system operators, and the disturbance factor $\delta_{w t}^{+}(w=i, j, \ldots, k)$ can be specified by system operators basing on the historical wind power data. Similarly, $z_{a t}^{-}$denotes the negative fluctuation of area $a$, and

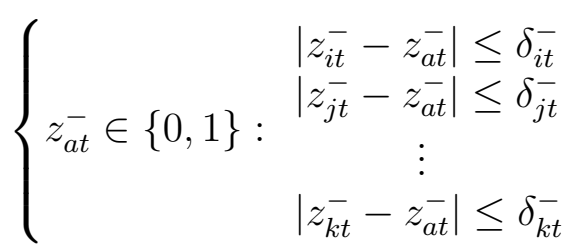

where $\delta_{w t}^{-}$is also a disturbance factor that determines the consistency of negative fluctuations, so as to maintain the synchronous volatility of multiple wind farms. It is noted that each absolute term in (8) and (9) can be easily converted into two linear inequalities, avoiding the nonlinear operations in the uncertainty set [35]. Specifically, the illustration of the enhanced polyhedron uncertainty set is display in Fig. 5. By introducing the linear cuts into the polyhedron uncertainty set $\mathcal{U}^{w}$, we formulate the enhanced polyhedron uncertainty set $\widehat{\mathcal{U}}^{w}$, which can eliminate the unnecessary uncertain scenarios and alleviate the conservatism of the robust UC model. In particular, the wind power generation is assumed to be independent of each other for the wind farms located in two distant areas. As shown in Fig. 4 , the wind power generations of wind farms $i, j, \ldots, k$ in the area $a$ have no bearing on the wind farms $m, n$ in the area $b$.

Remark 1. As indicated in [36], the worst case in $\mathcal{U}^{w}$ must be realized by the vertices of the polyhedron uncertainty set, that is, the power output of wind farms must equal to their lower bound, upper bound or forecast value. In this regard, the candidate value of variables $z_{w t}^{+}$ and $z_{w t}^{-}$must be either zero or one. To maintain the optimality of the proposed model, the disturbance factors $\delta_{w t}^{+}$and $\delta_{w t}^{-}$are chosen to value from the set $[0,1)$. To be more specific, when $\delta_{w t}^{+}$and $\delta_{w t}^{-}$is arbitrarily taken at $[0,1)$, the result does not make any difference since 


$$
\begin{aligned}
& \hat{\mathcal{U}}^{w}=\left\{p_{w t}^{\mathrm{w}_{(-)}}, \forall w \in W, t \in \mathcal{T} \mid\right. \\
& p_{w t}^{\mathrm{W}_{a}}=\tilde{p}_{w t}^{\mathrm{W}_{a}}+\left(\bar{p}_{w t}^{\mathrm{W}_{a}}-\tilde{p}_{w t}^{\mathrm{W}_{a}}\right) \cdot z_{w t}^{+}-\left(\tilde{p}_{w t}^{\mathrm{W}_{a}}-\underline{p}_{w t}^{\mathrm{W}_{a}}\right) \cdot z_{w t}^{-}, \\
& \left.\left|z_{w t}^{+}-z_{a t}^{+}\right| \leq \delta_{w t}^{+},\left|z_{w t}^{-}-z_{a t}^{-}\right| \leq \delta_{w t}^{-}, z_{a t}^{+}\right) z_{a t}^{-}, \in\{0,1\}, \\
& z_{w t}^{+}, z_{w t}^{-} \in\{0,1\}, \forall w \in W_{a}, t \in \mathcal{T}, \\
& \vdots \quad \vdots \quad \vdots \\
& p_{w t}^{\mathrm{W}_{b}}=\tilde{p}_{w t}^{\mathrm{W}_{b}}+\left(\bar{p}_{w t}^{\mathrm{W}_{b}}-\tilde{p}_{w t}^{\mathrm{W}_{b}}\right) \cdot z_{w t}^{+}-\left(\tilde{p}_{w t}^{\mathrm{W}_{b}}-\underline{p}_{w t}^{\mathrm{W}_{b}}\right) \cdot z_{w t}^{-}, \\
& \left.\left|z_{w t}^{+}-z_{b t}^{+}\right| \leq \delta_{w t}^{+}, \quad\left|z_{w t}^{-}-z_{b t}^{-}\right| \leq \delta_{w t}^{-}, z_{b t}^{+}\right), z_{b t}^{-}, \in\{0,1\}, \\
& z_{w t}^{+}, z_{w t}^{-} \in\{0,1\}, \forall w \in W_{b}, t \in \mathcal{T} \\
& \sum_{t \in \mathcal{T}}\left(z_{w t}^{+}+z_{w t}^{-}\right) \leq \Gamma_{w}, \sum_{w \in W}\left(z_{w t}^{+}+z_{w t}^{-}\right) \leq \Pi_{t}, \forall w \in W, W=W_{a} \cup \cdots \cup W_{b} \\
& \}
\end{aligned}
$$
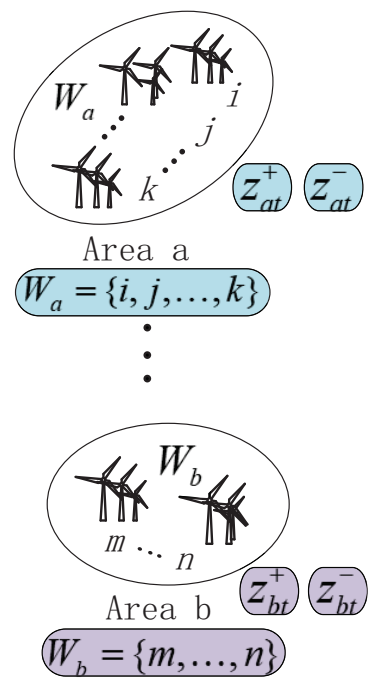

Figure 5: The illustration of the enhanced polyhedral uncertainty set

$z_{w t}^{+}$and $z_{w t}^{-}$must be either zero or one. As a result, for the sake of simplicity, the disturbance factors are set as 0.5 in this paper.

Remark 2. In the enhanced uncertainty set, the correlation of adjacent wind farms in one area is taken into consideration by introducing regional fluctuation variables that characterize the synchronous volatility of wind farms. When each wind farm is located in its own area and independent of each other, the enhanced uncertainty set will degenerate into a traditional uncertainty set.

\section{The data-adaptive robust unit commitment model}

\subsection{Problem formulation of robust unit commitment}

In this paper, a data-adaptive robust unit commitment (DARUC) model under high penetration of wind power is proposed. The robust UC problem is formulated as a two-stage risk-averse unit commitment model, among which the first stage problem determines the day-ahead commitment decisions $\boldsymbol{y}$ for the minimal commitment cost within the planning horizon, and the second stage solves economic dispatch problems after the realization of uncertain wind power generation, it is formulated as follow: 


$$
\begin{array}{ll}
\min _{\boldsymbol{y}} \sum_{t \in \mathcal{T}} \sum_{i \in \mathcal{G}}\left(S_{i}^{U} u_{i t}+S_{i}^{D} v_{i t}\right)+\max _{\boldsymbol{u}^{\boldsymbol{w}} \in \widehat{\mathcal{U}}^{w}} \min _{\boldsymbol{x} \in \Omega\left(\boldsymbol{y}, \boldsymbol{u}^{\boldsymbol{w}}\right)} \sum_{t \in \mathcal{T}}\left\{\sum_{i \in \mathcal{G}} C_{i}\left(p_{i t}\right)\right. \\
\\
\\
\text { s.t. } \left.\quad-\sum_{d \in \mathcal{D}} c_{d t} \Delta p_{d t}^{\mathrm{D}}+\sum_{w \in \mathcal{W}} c_{w t} \Delta p_{w t}^{\mathrm{W}}\right\} \\
\quad \forall i \in \mathcal{G}, \forall t \in \mathcal{T}, 1 \leq k-(t-1) \leq M U_{i}, \\
\quad y_{i(t-1)}-y_{i t}+y_{i k} \leq 1, \\
\quad \forall i \in \mathcal{G}, \forall t \in \mathcal{T}, 1 \leq k-(t-1) \leq M D_{i} \\
\quad-y_{i(t-1)}+y_{i t}-u_{i t} \leq 0, \quad \forall i \in \mathcal{G}, \forall t \in \mathcal{T} \\
& y_{i(t-1)}-y_{i t}-v_{i t} \leq 0, \quad \forall i \in \mathcal{G}, \forall t \in \mathcal{T} \\
& y_{i t}, u_{i t}, v_{i t} \in\{0,1\}, \quad \forall i \in \mathcal{G}, \forall t \in \mathcal{T}
\end{array}
$$

where $\boldsymbol{y}$ includes the binary variables $y_{i t}, u_{i t}$ and $v_{i t}, y_{i t}$ represents the on/off status of generator $i$ at time $t, u_{i t}$ and $v_{i t}$ indicate whether generator $i$ turns on/shuts down at time $t$, respectively, $\mathcal{G}$ denotes the set of generator units, $\boldsymbol{u}^{\boldsymbol{w}}$ is the random wind power generation that characterized by the enhanced uncertainty set $\widehat{\mathcal{U}}^{w}$, and $S_{i}^{U}, S_{i}^{D}$ respectively represent the startup cost and shutdown cost of generator $i . \Delta p_{w t}$ and $\Delta p_{d t}^{\mathrm{D}}$ represent the wind power curtailment and the load shedding in period $t$, respectively, $c_{w t}$ and $c_{d t}$ are the corresponding penalty coefficient. $M U_{i}$ and $M D_{i}$ are the minimum up/down time of generator $i$, equations (11) and (12) represent the minimum up/down time constraints of generation units, and the logic constraints of generator unit status are described in (13) and (14).

Specifically, $\Omega\left(\boldsymbol{y}, \boldsymbol{u}^{\boldsymbol{w}}\right)$ defines the feasible set of the second-stage decision variables $\boldsymbol{x}$ for a fixed commitment decision $\boldsymbol{y}$ and wind power generation $\boldsymbol{u}^{\boldsymbol{w}}$, which can be further expressed as: 


$$
\begin{aligned}
& p_{i t}+s_{i t} \leq y_{i t} P_{i}^{\max }, \quad \forall i \in \mathcal{G}, \forall t \in \mathcal{T} \\
& p_{i t} \geq y_{i t} P_{i}^{\min }, \quad \forall i \in \mathcal{G}, \forall t \in \mathcal{T} \\
& p_{i(t-1)}-p_{i t} \leq R D_{i} \cdot y_{i t}+P_{i}^{\max } \cdot\left(1-y_{i t}\right), \quad \forall i \in \mathcal{G}, \forall t \in \mathcal{T} \\
& p_{i t}-p_{i(t-1)}+s_{i t} \leq R U_{i} \cdot y_{i(t-1)}+P_{i}^{\max } \cdot\left(1-y_{i(t-1)}\right), \quad \forall i \in \mathcal{G}, \quad \forall t \in \mathcal{T} \\
& 0 \leq s_{i t} \leq C R_{i} \cdot y_{i t}, \quad \forall i \in \mathcal{G}, \forall t \in \mathcal{T} \\
& 0 \leq o_{i t} \leq s_{i t}+C Q_{i}\left(1-y_{i t}\right), \quad \forall i \in \mathcal{G}, \forall t \in \mathcal{T} \\
& \sum_{i \in \mathcal{G}} s_{i t} \geq S_{t}, \quad \forall t \in \mathcal{T} \\
& \sum_{i \in \mathcal{G}} o_{i t} \geq O_{t}, \quad \forall t \in \mathcal{T} \\
& \sum_{i \in \mathcal{G}} p_{i t}+\sum_{w \in \mathcal{W}}\left(p_{w t}^{\mathrm{W}}-\Delta p_{w t}^{\mathrm{W}}\right)=\sum_{d \in \mathcal{D}}\left(p_{d t}^{\mathrm{D}}-\Delta p_{d t}^{\mathrm{D}}\right), \quad \forall t \in \mathcal{T} \\
& -F_{i j}^{\max } \leq \sum_{b \in \mathcal{B}} K_{i j}^{b}\left(\sum_{i \in \mathcal{G}_{b}} p_{i t}+\sum_{w \in \mathcal{W}_{b}}\left(p_{w t}^{\mathrm{W}}-\Delta p_{w t}^{\mathrm{W}}\right)-\sum_{d \in \mathcal{D}_{b}}\left(p_{d t}^{\mathrm{D}}-\Delta p_{d t}^{\mathrm{D}}\right)\right) \leq F_{i j}^{\max },
\end{aligned}
$$

where $p_{i t}$ is the power generator of generator $i$ at time period $t, P_{i}^{\max }$ and $P_{i}^{\min }$ represent the maximum and minimum power generation of generator $i$, respectively, $s_{i t}$ is the spinning reserve of generator $i$ in time $t$, and it is restricted by $C R_{i}$, the ramping capacity of generator $i$, $o_{i t}$ denotes the operating reserve of generator $i$ in time $t$ and $C Q_{i}$ is the quick start capacity of generator $i, S_{t}$ and $O_{t}$ represent the spinning and operating reserve requirement in time $t$, respectively, $R D_{i}$ and $R U_{i}$ are respectively the ramp-down/ramp-up rate limit for generator $i$. $\mathcal{D}$ and $\mathcal{B}$ denote the sets of load and bus, respectively, $p_{d t}^{\mathrm{D}}$ denotes the load of $d$ in time $t, F_{i j}^{\max }$ is the capacity for transmission line connecting bus $i$ and $j$, and $K_{i j}^{b}$ represents the distribution factor for the transmission line due to the net injection at bus $b, \mathcal{L}$ is the set of all transmission lines. For the second stage problem, (16) and (17) are the maximum and minimum generation output constraints of generators that couple the commitment and dispatch decision, (18) - (25) include dispatch related constraints such as ramping constraints, reserve requirement, power balance and transmission limits, respectively.

It should be noted that the quadratic function of generation $\operatorname{cost} C_{i}(\cdot)$ can be approximated by a piecewise linear function with $n$ pieces [37], as described:

$$
C_{i}\left(p_{i t}\right)=\psi_{i t} \geq \gamma_{i}^{n} y_{i t}+\nu_{i}^{n} p_{i t}
$$

where $\gamma_{i}^{n}$ and $\nu_{i}^{n}$ represent the intercept and slope of the $n^{\text {th }}$ linear piece in the piecewise linear approximation for the $i^{\text {th }}$ generator. 


\subsection{Solution Methodology}

For simplicity, the two-stage DARUC model can be written in a compact matrix form as follows:

$$
\min _{\boldsymbol{y} \in \Omega^{\boldsymbol{F}}} \boldsymbol{c}^{\mathrm{T}} \boldsymbol{y}+\max _{\boldsymbol{u}^{\boldsymbol{w}} \in \widehat{\mathcal{U}}^{w}} \min _{\boldsymbol{x} \in \Omega\left(\boldsymbol{y}, \boldsymbol{u}^{\boldsymbol{w}}\right)} \boldsymbol{b}^{\mathrm{T}} \boldsymbol{x}
$$

where

$$
\begin{aligned}
& \Omega^{\boldsymbol{F}}=\{\boldsymbol{y} \mid \boldsymbol{A} \boldsymbol{y} \geq \boldsymbol{d}\}, \\
& \Omega\left(\boldsymbol{y}, \boldsymbol{u}^{\boldsymbol{w}}\right)=\left\{\boldsymbol{x} \mid \boldsymbol{G} \boldsymbol{x} \geq \boldsymbol{h}-\boldsymbol{E} \boldsymbol{y}-\boldsymbol{M} \boldsymbol{u}^{\boldsymbol{w}}\right\}
\end{aligned}
$$

where (28) represents all constraints in the first stage (11)-(15) and (29) refers to the constrains of the second stage (16)-(25). Here, the first-stage decisions $\boldsymbol{y}$ should be robust to any realization of the wind scenarios $\boldsymbol{u}^{\boldsymbol{w}}$ in the enhanced uncertainty set $\widehat{\mathcal{U}}^{w}$.

The C\&CG algorithm is proven to achieve an efficient solution for a mixed-integer linear programming problem [28]. In this paper, the two-stage DARUC model is decomposed into a master problem (MP) and a subproblem (SP) with uncertain wind power scenarios, and the $\mathrm{C} \& \mathrm{CG}$ framework is adopted to address the proposed model by iteratively solving the $\mathrm{MP}$ and the SP. To this end, the optimal solution of SP is regarded as a significant scenario and new variables and corresponding constraints will be generated and added to the MP in each iteration. More specifically, the procedure of the C\&CG algorithm is elaborately presented in Fig. 6.

Remark 3. The max-mix structure in the subproblem can be converted into a single-level maximum optimization problem based on the strong duality theory. It is noted that the bilinear term occurred in the new maximum objective function can be solved efficiently using the big-M linearization method, which can be referred to [38] for more detailed information.

\section{Numerical simulations}

To validate the effectiveness of the proposed data-adaptive robust unit commitment model, we divide the numerical simulations into two parts in this paper. In the first one, a statistical analysis of the three adjacent wind farms with a rated capacity of $90 \mathrm{MW}$ in Hainan Province is performed to demonstrate the benefit of copula function in obtaining the lower and upper boundaries of wind power generation. In the second one, numerical experiments are conducted out on a modified IEEE-118 bus system to verify the superiority of the enhanced uncertainty set [39]. The wind power outputs with 1-hour resolution from the wind farms in Hainan Province is taken account to obtain the data-adaptive wind power generation. Five wind farms are connected to the bus 2 and 7 in area 1, as well as the bus 78, 97, and 102 in area 2, respectively. The simulations are conducted on a computer with Intel(R) Core(TM) i7-4770 CPU @ 3.40GHz. The DARUC optimization is implemented in MATLAB and solved by GUROBI 9.0, and the optimality tolerance gap for the SP and MP is set as $10^{-4}$ in this paper. 


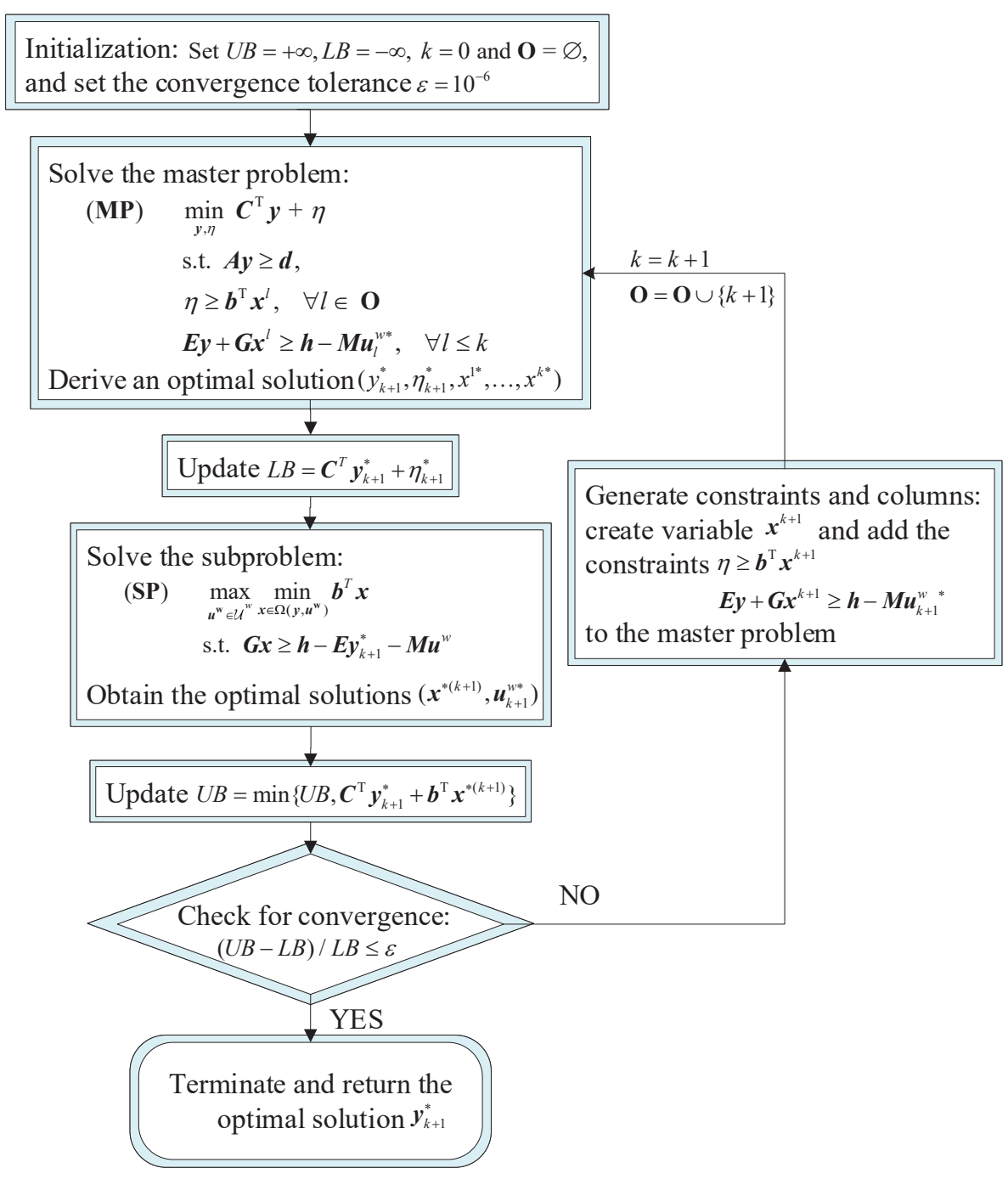

Figure 6: The solution procedure of $\mathrm{C} \& \mathrm{CG}$

\subsection{Analysis of the data-adaptive wind power generation}

\subsubsection{The validation of the t-copula function for the joint distribution function}

In this part, the 1-hour resolution wind power data of three adjacent wind farms in Hainan Province from 2014 to 2015 are selected for analysis [27]. Here, the t-copula function is applied in this paper to capture the correlation of wind power among multiple wind farms, and the analysis of reliability for the adoption of t-copula is presented in Appendix B. As the scatter plot of three wind farms shown in Fig. 7, the principal diagonals of both graphs (a) and (b) have the same distributions, which correspond to the marginal distribution functions of the three wind farms \#1, \#2 and \#3, respectively. The other six non-diagonal elements of graphs (a) and (b) display the scatterplot of wind power generation between the two corresponding wind farms, which can visually illustrate the correlation between the two 


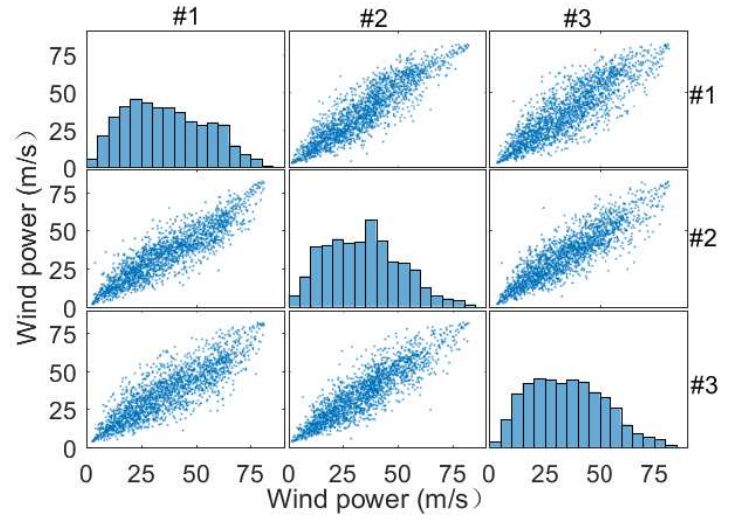

(a) Considering the correlation

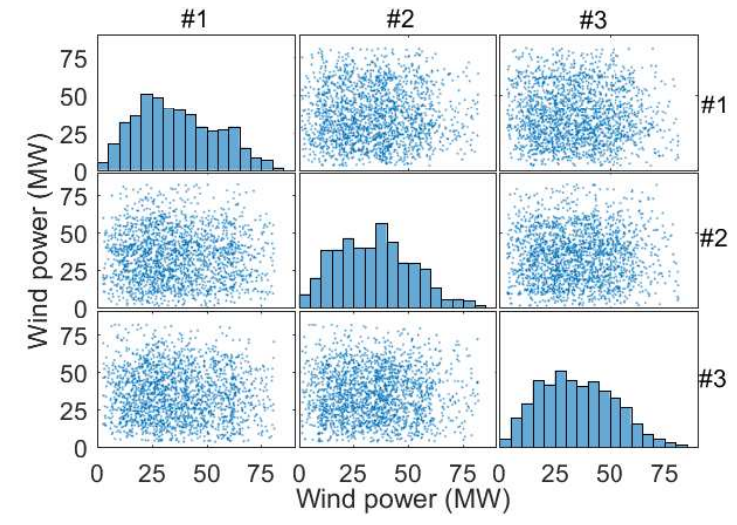

(b) Ignoring the correlation

Figure 7: The generated wind power samples of three adjacent wind farms

wind farms. For the graph (a), the t-copula function is applied to capture the correlation of the three wind farms for each time period from the historical wind power data, and the obtained joint distribution function is used to generate the uncertain wind power samples based on the quasi-Monte Carlo simulations method. At the same time, the wind power samples neglecting the correlation of wind farms are generated for comparison as shown in (b), among which the power output of three wind farm is independent of each other, but follows their respective marginal distribution.

To reflect the difference of the wind power samples (a) and (b) in Fig. 7 more intuitively, the measure function $\lambda(r)$ proposed in [40] that provides a visual degree of closeness is given as:

$$
\begin{aligned}
& K(r)=\operatorname{Pr}\left(C\left(u_{1}, u_{2} \ldots, u_{n}\right) \leq r\right) \\
& \lambda(r)=r-K(r)
\end{aligned}
$$

where $u_{i}, i=1,2, \ldots, n$ is the marginal distribution function of the $i^{\text {th }}$ random variable and $C(\cdot)$ represents the multivarite cumulative distribution function (CDF). As referred to Appendix A, we define the empirical $\operatorname{CDF} \hat{C}_{M}\left(u_{1}, u_{2}, u_{3}\right)$ based on the available historical wind power data, which can be used to evaluate the fitting effect of the proposed t-copula function as well as those ignores the correlation. Therefore, the lambda function $\lambda(r)$ with respect to the historical data of the empirical CDF, the wind power scenarios (a) of the tcopula function and the wind power scenarios (b) of ignoring the correlation are respectively calculated out, as illustrated in Fig. 8. It can be seen from the figure that the curve obtained from the wind power scenarios (a) of t-copula function is remarkably similar to the empirical CDF of historical data, while the curve obtained from the CDF of wind power scenarios (b) differ greatly from the former two. Although the wind power outputs of three wind farms follow their respective marginal distributions, the wind power scenarios (b) are independent of each other and cannot maintain the characteristics of the historical data, which explains the great difference between the green line and the empirical CDF. On the contrary, the 


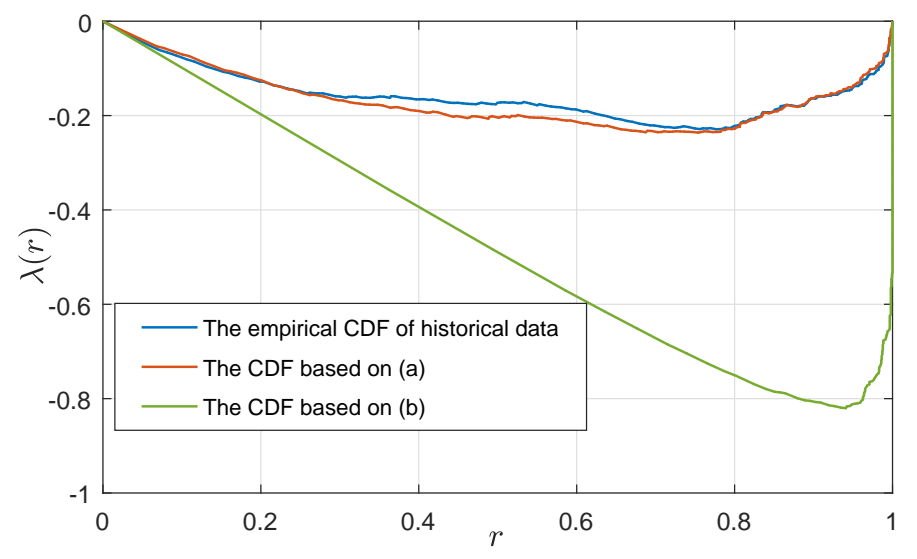

Figure 8: The comparison of lambda function $\lambda(r)$

wind power scenarios (a) not only follow the marginal distribution of each wind farm, but also maintain the spatial correlation of the three wind farms. In this respect, the generated wind power scenarios of t-copula function are more consistent with the historical wind power samples, which indicates that the t-copula function applied in this paper can well describe the correlation of multivariable and perfectly formulate the joint probability distribution.

\subsubsection{The benefit of $t$-copula function for the IDM model}

Consequently, with the available wind power scenarios (a), the imprecise Dirichlet model (IDM) [32] is applied to derive the bounds of CDF, which is illustrated in Fig. 9. We can find that when the number of wind power scenarios reaches 2000, the upper and lower bounds of $\mathrm{CDF}$ are quite close, among which the gap is within an acceptable range. However, when extracted from the available historical wind power samples directly, as the dotted lines shown in the figure, there is a large gap between the upper and lower bounds of CDF, which will lead to a large error in obtaining the boundaries of wind power generation.

It is observed that the historical data is insufficient to obtain the convergent CDF, with the number of 700 for each time period (resolution of 1-hour from 2014 to 2015). However, by applying the copula theory, we can generate as many wind power scenarios as possible that maintain the characteristic of historical data. These wind power scenarios are then used to obtain the convergent CDF through the IDM method. Therefore, we can effectively improve the applicability of the IDM method by the introduction of copula function. Afterwards, the data-adaptive wind power generation can be determined from the obtained CDF with a given confidence level.

\subsubsection{The necessity for considering correlation among multiple wind farms}

Here, we further illustrate the necessity for considering the correlation among multiple wind farms. Rather than assuming that the lower and upper bounds of wind power generation are specific values, this paper determines the boundaries of wind power generation based on the available wind power scenarios. Firstly, the wind power generation of three 


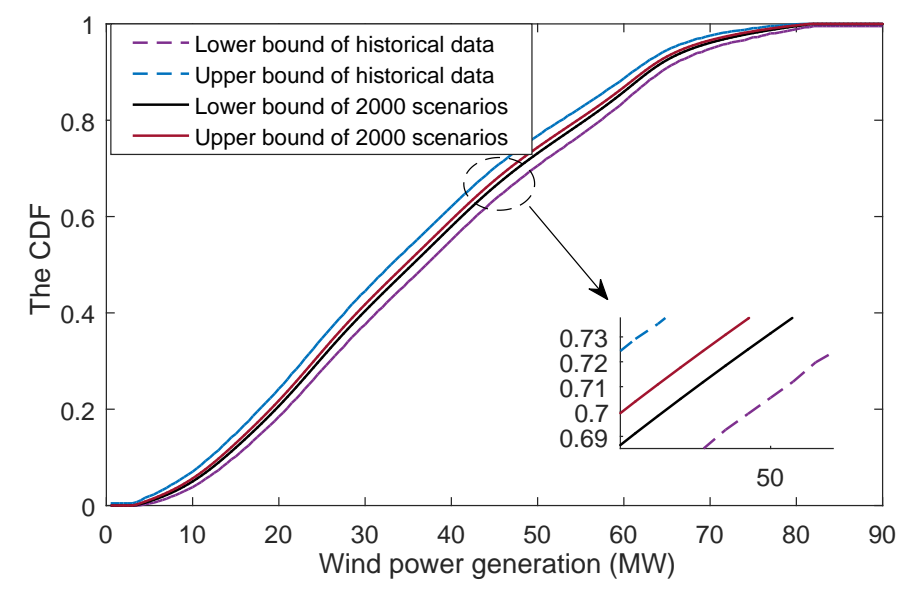

Figure 9: The bounds of CDF based on the imprecise Dirichlet model

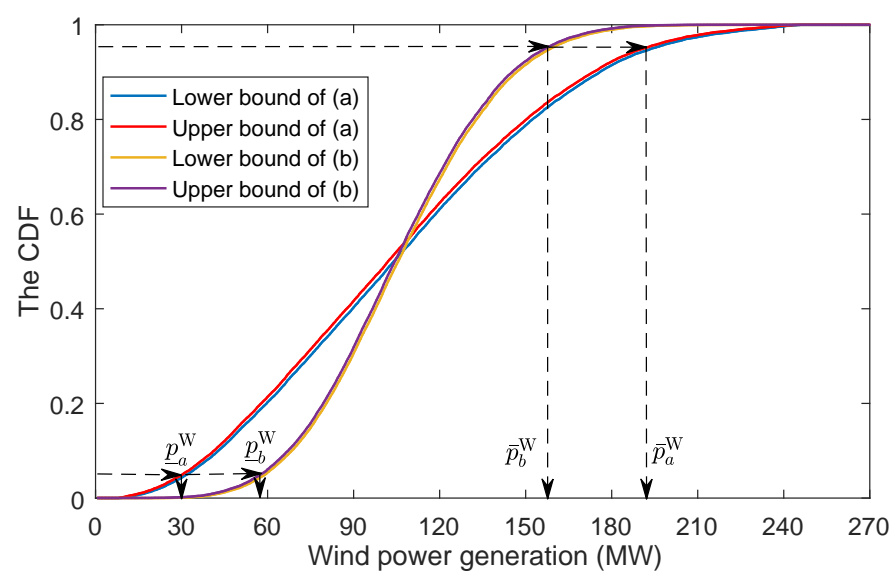

Figure 10: The data-adaptive wind power generation

wind farms is figured out through the IDM method basing on the wind power scenarios (a) and (b) of Fig. 7, respectively. As shown in Fig. 10, with the number of scenarios being 2000 , both the convergent CDFs of (a) and (b) are derived, and the wind power generation $\left[\underline{p}_{a}^{\mathrm{W}}, \bar{p}_{a}^{\mathrm{W}}\right]$ and $\left[\underline{p}_{b}^{\mathrm{W}}, \bar{p}_{b}^{\mathrm{W}}\right]$ respectively equal to $[30.7 \mathrm{MW}, 192.3 \mathrm{MW}]$ and $[57.2 \mathrm{MW}, 157.5$ MW] when the confidence level is set as $95 \%$. Obviously, the fluctuant range of wind power is underestimated when the correlation of wind farms is ignored, since the power output of adjacent wind farms tends to be dependent in actual situations. In this regard, the dispatch solution will become impractical when the boundaries of wind power generation, $\left[p_{b}^{\mathrm{W}}, \bar{p}_{b}^{\mathrm{W}}\right]$, are applied to construct the polyhedron uncertainty set. In contrast, the data-adaptive wind power generation $\left[\underline{p}_{a}^{\mathrm{W}}, \bar{p}_{a}^{\mathrm{W}}\right]$ is more accurate due to the consideration of correlation among multiple wind farms, which objectively reflects the available information, and will ultimately lead to a more reliable polyhedron uncertainty set. 


\subsection{Analysis of the risk-averse unit commitment model}

\subsubsection{Simulations under low wind power penetration}

In this section, the data-adaptive wind power generations of 24 time periods based on the first part are directly used to evaluate the effectiveness of the DARUC model. Here, we aim to verify the superiority of the enhanced uncertainty set over the traditional uncertainty set, with the penetration of wind power being $10 \%$. The installed capacity of each wind farm in area 1 and area 2 are set as $150 \mathrm{MW}$ and $90 \mathrm{MW}$, respectively. To illustrate the validity of the proposed approach, the following three cases are studied.

Case 1: The enhanced uncertainty set $\widehat{\mathcal{U}}^{w}$ is incorporated into the robust UC model, and the wind farms in each area are correlated while the wind farms in different areas are uncorrelated;

Case 2: The traditional uncertainty set $\mathcal{U}^{w}$ is incorporated into the robust UC model, and the five wind farms in the 2 areas are considered uncorrelated;

Case 3: The enhanced uncertainty set $\widehat{\mathcal{U}}^{w}$ is incorporated into the robust UC model, and the five wind farms are considered correlated.

Table 1: Operation results under different cases with low wind power penetration

\begin{tabular}{ccccc}
\hline Index & TC $(\$)$ & SC $(\$)$ & OC $(\$)$ & Iteration \\
\hline case 1 & 1469105 & 463674.3923 & 1005430.7481 & 2 \\
case 2 & 1477883 & 466576.0822 & 1011307.5522 & 3 \\
case 3 & 1423854 & 451036.3821 & 972817.7908 & 2 \\
\hline
\end{tabular}

Table 1 compares the operation costs of the 3 cases with the low wind power penetration, including the total cost (TC), start-up cost of units (SC), and the operational cost (OC), as well as the number of iteration. It can be seen that the TC of case 1 is of $1469105 \$$, which is nearly $10000 \$$ lower than that of case 2 . More specifically, the SC of both cases is close to $465000 \$$, while the $\mathrm{OC}$ of case 2 is much higher than the OC of case 1 . This is because the synchronous volatility of wind farms in adjacent wind farms is taken into account in the case 1 , whereas the wind farms are considered uncorrelated in case 2 , so some unnecessary extreme scenarios are eliminated in $\widehat{\mathcal{U}}^{w}$, but they are included in $\mathcal{U}^{w}$ and lead to the more conservative results of case 2. In Fig. 11, the number of generator units that are on status for each time periods are displayed on the bar graph, from which we can find that case 1 and 2 have the similar unit commitment decision, which just coincides with the result that case 1 and case 2 have the similar SC.

As the comparison between case 1 and case 3 showed in Table 1, when more wind farms are involved, the synchronous volatility has a greater impact on the scheduling results. Similar results can be found in Fig. 11, when the five wind farms are correlated, they tend to reach their upper or lower bounds at the same time. As a result, more unnecessary extreme scenarios are excluded in the enhanced uncertainty set, which undoubtedly makes the final scheduling decision far less conservative, with the TC being $1423854 \$$ in case 3 . 


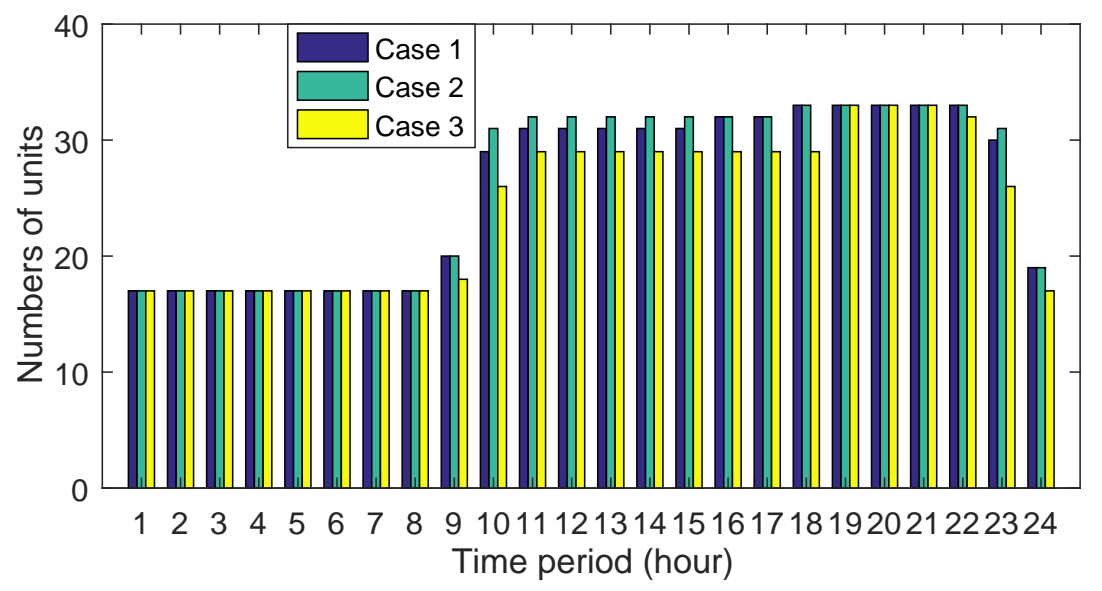

Figure 11: The number of on-status generator units under low wind power penetration

Furthermore, the out-of-sample Monte Carlo simulation is conducted to validate the commitment schedule. Here, the historical wind power data of one whole year (365 days) is applied to provide a fair comparison for the three cases. It should be pointed out that if the obtained commitment schedule can well adapt to the uncertain wind power generation, the operational cost of the realizations of wind power is no more than those in Table 1. That is because the unit commitment decision of generators is determined through the robust optimization that hedges against the worst-case scenarios within the constructed uncertainty set. In this respect, the smaller the gap between the actual OC and the worstcase OC, the less conservative of the robust UC model is, which means that the constructed uncertainty set is more in line with the actual situations. As illustrated in Fig. 12, the dotted red line represents the operational cost of the worst-case scenario in the process of robust optimization, which is equal to $1005430.7481(\$)$. It can be found that the realizations of historical wind power samples come close to the dotted red line, but never crossed the red line, which exactly validates the effectiveness of the proposed polyhedral uncertainty set. It can guarantee the robustness of the dispatch solution while alleviating the conservativeness of the robust UC model.

Compared with the results of case 1, Fig. 13 displays the operational cost of historical wind power samples under the commitment schedule of case 2, among which the correlation of adjacent wind farms is ignored. It can be observed that the green line representing the most terrible scenarios in actual situations is far less than the optimized worst-case scenarios in case 2 , which indicates that some extreme scenarios are included and aggravate the conservativeness of the robust UC model when neglecting the correlation among adjacent wind farms. Nevertheless, the commitment schedule of case 2 is at the cost of the economy, which can still guarantee the reliability of the system. On the contrary, although case 3 can obtain a much less conservative decision since many extreme scenarios are excluded by considering the correlation of the five wind farms in the 2 areas. However, it overestimates the correlation of wind farms due to the fact that the wind farms in the two distant areas are irrelevant. As a result, we can find from Fig. 14 that the dotted red line representing 


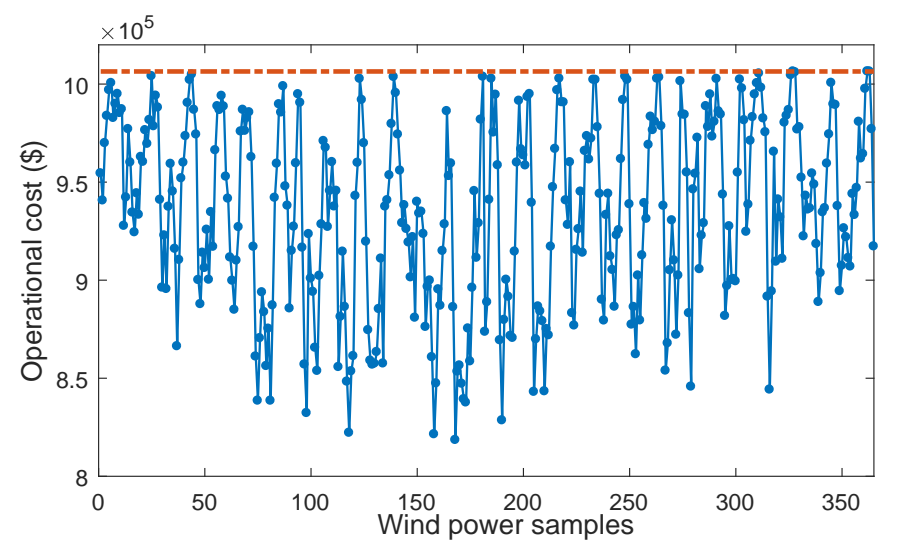

Figure 12: The realizations of historical wind power samples under the commitment schedule of case 1

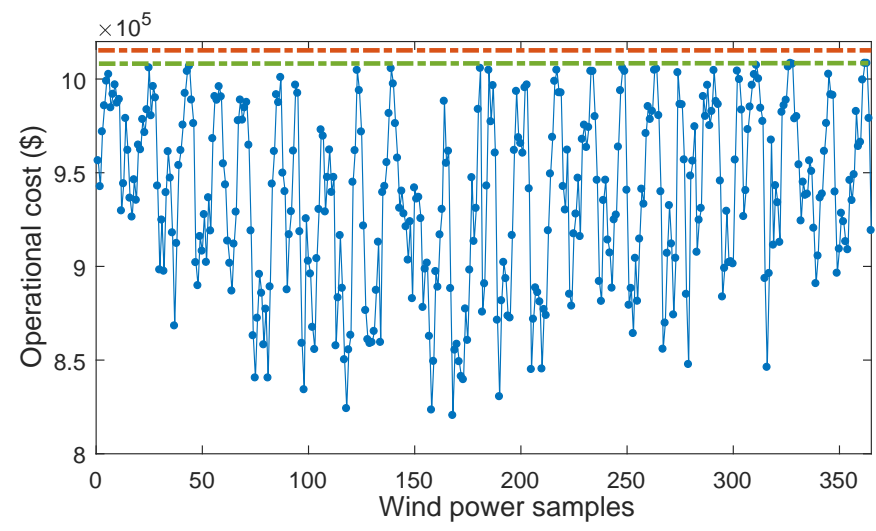

Figure 13: The realizations of historical wind power samples under the commitment schedule of case 2

the worst-case scenario in the constructed uncertainty set has greatly underestimated the fluctuation of wind power generation, and the load shedding and wind power curtailment have to be implemented to ensure the robustness of the system, which explains the maximum operational cost of actual samples represented by the green line is much higher than the red line.

Consequently, the comparisons of three cases verify the effectiveness of the enhanced uncertainty set, and the proper construction of uncertainty set can greatly alleviate the conservativeness of the DARUC model. By maintaining the synchronous volatility trend of the adjacent wind farms, the enhanced uncertainty set is more in line with the actual situations, which helps to derive the more practical scheduling decision for the UC problem. It is noted that the traditional uncertainty set $\mathcal{U}^{w}$ in case 2 can be recognized as the five wind farms locating in five areas in the enhanced uncertainty set $\widehat{\mathcal{U}}^{w}$, which can further illustrate the generality of the proposed DARUC model. 


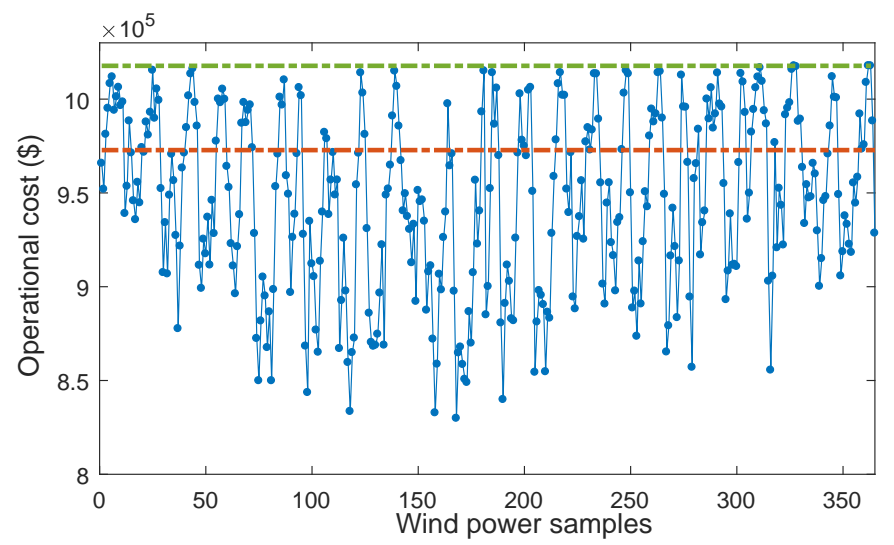

Figure 14: The realizations of historical wind power samples under the commitment schedule of case 3

Table 2: Operation results under different cases with high wind power penetration

\begin{tabular}{ccccc}
\hline Index & TC (\$) & SC $(\$)$ & OC $(\$)$ & Iteration \\
\hline case 1' & 1391085 & 401949.9496 & 993548.7492 & 3 \\
case 2' & 1421171 & 438771.2721 & 981579.1044 & 4 \\
case 3' & 1323780 & 422492.8219 & 901287.0504 & 2 \\
\hline
\end{tabular}

\subsubsection{Simulations under high wind power penetration}

To make the proposed DARUC model more convincing, more case studies are conducted on the modified IEEE-118 bus system with the installed capacity of each wind farms being $300 \mathrm{MW}$. In this regard, wind power with an installed capacity $1500 \mathrm{MW}$ will eventually lead to the wind power penetration of power systems at a level of about $25 \%$, which is in line with the current background of large-scale renewable energy integrating into the power system. Similar to the setting of the three former case studies, the three new cases are denoted as case 1', case 2' and case 3', respectively.

The experimental results of different cases can be found in Table 2. Obviously, when the penetration of wind power increases, the difference of the scheduling results in three cases becomes more significant. On the one hand, when high penetration of wind power into the power systems is considered, the unnecessary extreme scenarios lead to over-conservative scheduling results, which is impractical in actual operation, as the comparison of case 1' and case 2' shown in the table. In other words, the two wind farms in area 1 and the three wind farms in area 2 are respectively correlated in reality, which should be considered when deriving the optimal scheduling solution. However, case 2' ignores the characteristic of synchronous volatility in adjacent wind farms, and its scheduling results are bound to be over-conservative. Similarly, Fig. 15 illustrates the number of the on-status generator units under high penetration of wind power. It can be seen that the on-status generator units in case 1' is much less than those in case 2', so the case 1' has the less SC as 401949.9496 \$, 


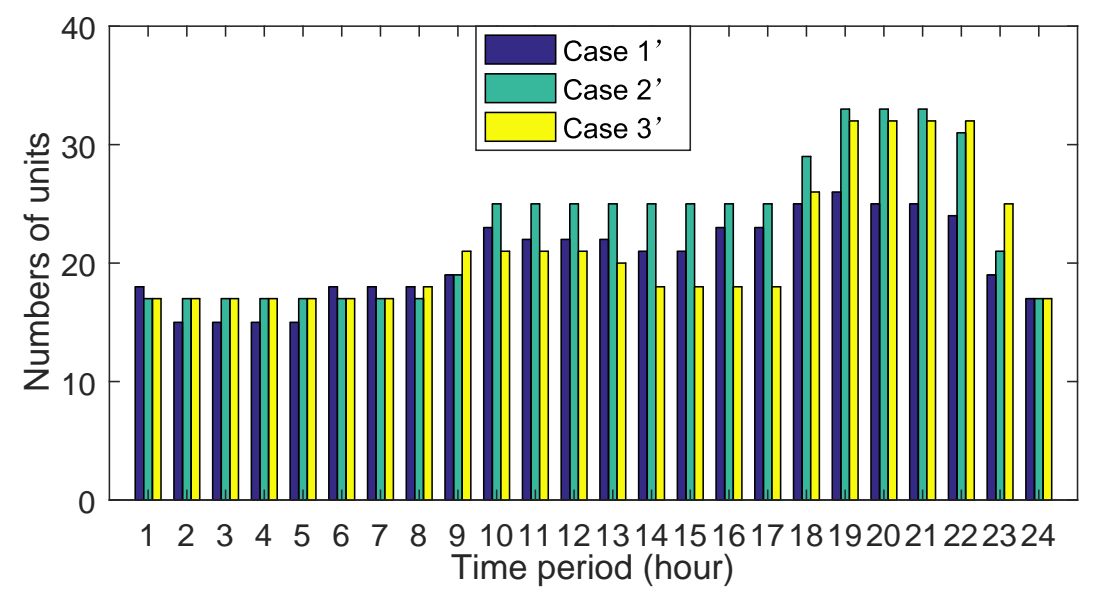

Figure 15: The number of on-status generator units under high wind power penetration

comparing to $438771.2721 \$$ in case 2'. Although the OC of case 1' is slightly higher than that of case 2', the TC of case 1' is nearly $30000 \$$ less than case 2', among which the value is only $10000 \$$ when in low wind power penetration. In this regard, the proposed DARUC model becomes more effective when exposed to higher wind power penetration.

On the other hand, the situation where more wind farms are involved in an area is also taken into consideration. For example, the five wind farms are featured in the synchronous volatility in case 3'. It is observed from Table 2 that the more adjacent wind farms drive the scheduling results of case 3' to be better than those in case 1'. Due to more unnecessary extreme scenarios are eliminated in the enhanced uncertainty set $\widehat{\mathcal{U}}^{w}$, and fewer extreme scenarios emerge in the uncertainty set, which leads to the more practical result when the proposed DARUC model is applied.

\section{Conclusion}

With high-level wind power generation penetrating into the power systems, the riskaware data-adaptive robust unit commitment (DARUC) model is proposed in this paper. We explore the random characteristics of wind power from the available wind power data and incorporate the statistical information into the traditional robust optimization model. Here, the correlation among different wind farms is investigated by the copula theory and the imprecise Dirichlet model (IDM) is applied in this paper to derive the data-adaptive wind power generation, which helps to construct a more reliable polyhedron uncertainty set. Meanwhile, to eliminate the unnecessary extreme scenarios and alleviate the conservativeness of the robust scheduling model, the characteristic that the volatility in adjacent wind farms has a synchronous trend is fully considered in the enhanced polyhedron uncertainty set. Simulation results verify that the proposed DARUC model can acquire more practical scheduling solutions, which can provide valuable information for the system operators. Further, the regional fluctuation variables and the auxiliary variables $z_{w t}(w=i, j, \ldots, k)$ are set as binary variables in this paper for simplicity. The investigation of the characteristics 
of enhanced uncertainty sets when these variables are extended to continuous variables in the range of $[0,1]$ will be considered in our future research.

\section{Appendix A. The mathematical formulation for the empirical CDF}

With the $M$ observations of available historical wind power data for the $n$ wind farms, we can derive the empirical $\mathrm{CDF} \hat{C}_{M}\left(u_{1}, u_{2}, \ldots, u_{n}\right)$ based on the $n$ marginal distributions $u_{1} \sim u_{n}$ that calculated by the CDF transformation $\left(u_{i}=F_{i}\left(x_{i}\right), i=1, \ldots, n\right)$, which is elaborated described as:

$$
\begin{array}{r}
\hat{C}_{M}\left(u_{1}, u_{2}, \ldots, u_{n}\right)=\frac{1}{M} \sum_{j=1}^{M} \mathbb{I}_{\left[F_{1}\left(x_{1}^{j}\right) \leq u_{1}\right]} \mathbb{I}_{\left[F_{2}\left(x_{2}^{j}\right) \leq u_{2}\right]} \cdots \mathbb{I}_{\left[F_{n}\left(x_{n}^{j}\right) \leq u_{n}\right]} \\
u_{1}, \ldots, u_{n} \in[0,1]
\end{array}
$$

where the indicator function is defined as follows:

$$
\mathbb{I}_{\left[F_{k}\left(x_{k}^{j}\right) \leq u_{k}\right]}= \begin{cases}1 & \text { if } F_{k}\left(x_{k}^{j}\right) \leq u_{k} \\ 0 & \text { else }\end{cases}
$$

Therefore, we can evaluate the fitting effect of the proposed t-copula function by estimating the Euclidean distance between the empirical CDF and the formulated t-copula function, which is expressed as:

$$
d_{t}^{2}=\sum_{i=1}^{M}\left|C^{t}\left(u_{1}^{i}, u_{2}^{i}, u_{3}^{i} ; \rho, k\right)-\hat{C}_{M}\left(u_{1}^{i}, u_{2}^{i}, u_{3}^{i}\right)\right|^{2}
$$

\section{Appendix B. The analysis of reliability for the adoption of t-copula function}

Here, we test the t-copula function with 1000 data samples of three random variables, and the experiments are divided into 6 groups, among which Test 1 is with the whole data samples, Test 2 is with the $1 \sim 800$ data samples, Test 3 is with the $1 \sim 600$ data samples, Test 4 is with the $1 \sim 400$ data samples, Test 5 is with the $1 \sim 200$ data samples, and finally Test 6 is with the $1 \sim 100$ data samples. Meanwhile, Test 7 is carried out to make a fair comparison between the Gaussian copula and t-copula. As shown in Table B.3, the evaluation of different tests includes the Euclidean distance (A.3) as well as the parameters of Copula model in terms of the Kendall coefficient and Spearman coefficient. It can be observed from Test 1 to Test 6 that the Kendall (Spearman) coefficient gradually converges to those of Test 1 as more data samples are involved, and the Euclidean distance of them is decreasing from 0.5879 to 0.1944 with the increasing data samples. As referred [30], the Euclidean distance of 0.1944 indicates that the t-copula function of Test 1 has a good fitting effect when characterizing the dependence structure of multiple variables. Meanwhile, the t-copula model of Test 3 has a Euclidean distance of 0.2440 , which is a low measure that can be tolerated by practical applications. Therefore, the 2 years of wind power data 
Table B.3: Results of correlation measure coefficient and Euclidean distance

\begin{tabular}{|c|c|c|c|c|c|c|c|}
\hline Index & \multicolumn{3}{|c|}{ Kendall coefficient } & \multicolumn{2}{|c|}{ Spearman coefficient } & \multicolumn{2}{|c|}{ Euclidean distance } \\
\hline Test $1(1: 1000)$ & $\begin{array}{c}1 \\
0.7067 \\
0.6039\end{array}$ & $\begin{array}{c}0.7067 \\
1 \\
0.6286\end{array}$ & $\begin{array}{c}0.6039 \\
0.6286 \\
1\end{array}$ & $\begin{array}{c}1 \\
0.8839 \\
0.7953\end{array}$ & $\begin{array}{c}0.8839 \\
1 \\
0.8185\end{array}$ & $\begin{array}{c}0.7953 \\
0.8185 \\
1\end{array}$ & 0.1944 \\
\hline Test $2(1: 800)$ & $\begin{array}{c}1 \\
0.6972 \\
0.5894\end{array}$ & $\begin{array}{c}0.6972 \\
1 \\
0.6217\end{array}$ & $\begin{array}{c}0.5894 \\
0.6217 \\
1\end{array}$ & $\begin{array}{c}1 \\
0.8839 \\
0.7814\end{array}$ & $\begin{array}{c}0.8767 \\
1 \\
0.8123\end{array}$ & $\begin{array}{c}0.7814 \\
0.8123 \\
1\end{array}$ & 0.2213 \\
\hline Test $3(1: 600)$ & $\begin{array}{c}1 \\
0.6942 \\
0.5831\end{array}$ & $\begin{array}{c}0.6942 \\
1 \\
0.6175\end{array}$ & $\begin{array}{c}0.5831 \\
0.6175 \\
1\end{array}$ & $\begin{array}{c}1 \\
0.8743 \\
0.7750\end{array}$ & $\begin{array}{c}0.8743 \\
1 \\
0.8083\end{array}$ & $\begin{array}{c}0.7750 \\
0.8083 \\
1\end{array}$ & 0.2440 \\
\hline Test $4(1: 400)$ & $\begin{array}{c}1 \\
0.6822 \\
0.5602\end{array}$ & $\begin{array}{c}0.6822 \\
1 \\
0.6058\end{array}$ & $\begin{array}{c}0.5602 \\
0.6058 \\
1\end{array}$ & $\begin{array}{c}1 \\
0.8656 \\
0.7526\end{array}$ & $\begin{array}{c}0.8656 \\
1 \\
0.7981\end{array}$ & $\begin{array}{c}0.7526 \\
0.7981 \\
1\end{array}$ & 0.3050 \\
\hline Test $5(1: 200)$ & $\begin{array}{c}1 \\
0.6712 \\
0.5228\end{array}$ & $\begin{array}{c}0.6712 \\
1 \\
0.5320\end{array}$ & $\begin{array}{c}0.5228 \\
0.5320 \\
1\end{array}$ & $\begin{array}{c}1 \\
0.8540 \\
0.7095\end{array}$ & $\begin{array}{c}0.8540 \\
1 \\
0.7196\end{array}$ & $\begin{array}{c}0.7095 \\
0.7196 \\
1\end{array}$ & 0.4392 \\
\hline Test $6(1: 100)$ & $\begin{array}{c}1 \\
0.7136 \\
0.5863\end{array}$ & $\begin{array}{c}0.7136 \\
1 \\
0.5954\end{array}$ & $\begin{array}{c}0.5863 \\
0.5954 \\
1\end{array}$ & $\begin{array}{c}1 \\
0.8627 \\
0.7715\end{array}$ & $\begin{array}{c}0.8627 \\
1 \\
0.7806\end{array}$ & $\begin{array}{c}0.7715 \\
0.7806 \\
1\end{array}$ & 0.5879 \\
\hline Test $7(1: 1000)$ & $\begin{array}{c}1 \\
0.6984 \\
0.6000\end{array}$ & $\begin{array}{c}0.6984 \\
1 \\
0.6250\end{array}$ & $\begin{array}{c}0.6000 \\
0.6250 \\
1\end{array}$ & {$\left[\begin{array}{c}1 \\
0.8839 \\
0.7954\end{array}\right.$} & $\begin{array}{c}0.8807 \\
1 \\
0.8189\end{array}$ & $\begin{array}{c}0.7954 \\
0.8189 \\
1\end{array}$ & 0.2137 \\
\hline
\end{tabular}

with a resolution of 1-hour is equipment to 730 data samples for each time period, which is absolutely sufficient for the t-copula function to capture the correlation of multivariate. Besides, the Gaussian-copula of Test 7 has a larger Euclidean distance than the t-copula of Test 1, which means the t-copula is much closer to the empirical copula and has a better fitting effect, and that is the reason for the adoption of t-copula in this paper.

Furthermore, the Kolmogorov-Smirnov statistics that measures the maximum absolute discrepancy between the Copula model and the empirical copula is also utilized to evaluate the goodness-of-fit in this paper [41]. As the boxplot shown in Fig. B.16, the first three tests (Test 1, 2, and 3) exhibit a low median Kolmogorov-Smirnov statistic, which indicates that the formulated t-copula with no less than 600 data samples is accurate enough to capture the dependence of random variables. Here, it is noted that the two years of wind power data is insufficient for the IDM method to derive the divergent confidence intervals. Instead, thousands of data samples are required for the IDM method, which exactly can be made up by the application of the Copula model. 


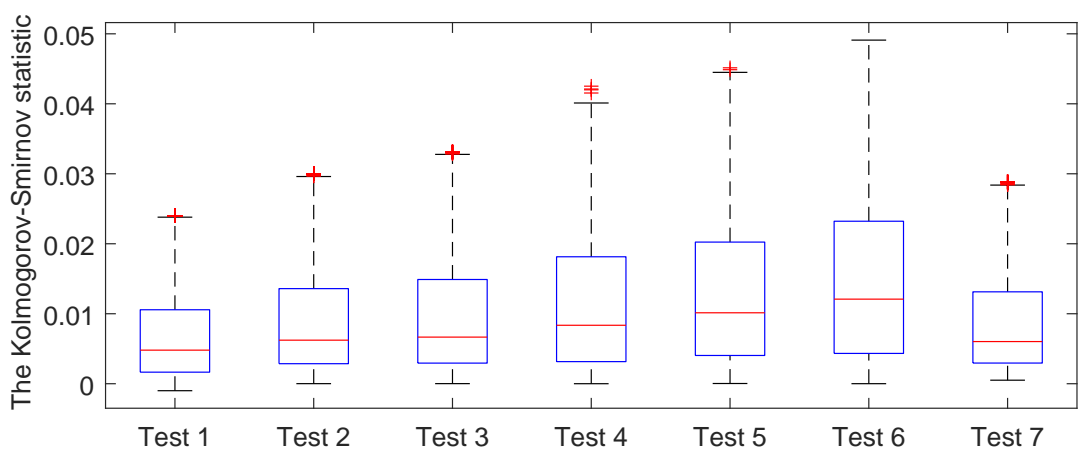

Figure B.16: The Kolmogorov-Smirnov statistic of the Copula model

[1] E. Cuce, D. Harjunowibowo, P. M. Cuce, Renewable and sustainable energy saving strategies for greenhouse systems: A comprehensive review, Renewable and Sustainable Energy Reviews 64 (2016) $34-59$.

[2] B. Kroposki, B. Johnson, Y. Zhang, V. Gevorgian, P. Denholm, B.-M. Hodge, B. Hannegan, Achieving a $100 \%$ renewable grid: Operating electric power systems with extremely high levels of variable renewable energy, IEEE Power and Energy Magazine 15 (2) (2017) 61-73.

[3] J. Li, S. Wang, L. Ye, J. Fang, A coordinated dispatch method with pumped-storage and battery-storage for compensating the variation of wind power, Protection and control of modern power Systems 3 (1) (2018) 2 .

[4] H. Chen, H. Ngan, Y. Zhang, Power System Optimization: Large-scale Complex Systems Approaches, John Wiley \& Sons, 2016.

[5] Y. Li, P. Wang, H. B. Gooi, J. Ye, L. Wu, Multi-objective optimal dispatch of microgrid under uncertainties via interval optimization, IEEE Transactions on Smart Grid 10 (2) (2017) 2046-2058.

[6] A. Shahirinia, E. Soofi, D. Yu, Probability distributions of outputs of stochastic economic dispatch, International Journal of Electrical Power \& Energy Systems 81 (2016) 308-316.

[7] S. Mohammadi, S. Soleymani, B. Mozafari, Scenario-based stochastic operation management of microgrid including wind, photovoltaic, micro-turbine, fuel cell and energy storage devices, International Journal of Electrical Power \& Energy Systems 54 (2014) 525-535.

[8] Y. Li, T. Zhao, P. Wang, H. B. Gooi, L. Wu, Y. Liu, J. Ye, Optimal operation of multimicrogrids via cooperative energy and reserve scheduling, IEEE Transactions on Industrial Informatics 14 (8) (2018) 3459-3468.

[9] P. Li, D. Yu, M. Yang, J. Wang, Flexible look-ahead dispatch realized by robust optimization considering cvar of wind power, IEEE Transactions on Power Systems 33 (5) (2018) 5330-5340.

[10] X. Li, Q. Zhai, Multi-stage robust transmission constrained unit commitment: A decomposition framework with implicit decision rules, International Journal of Electrical Power \& Energy Systems 108 (2019) 372-381

[11] D. Bertsimas, M. Sim, The price of robustness, Operations research 52 (1) (2004) 35-53.

[12] Z. Liang, H. Chen, X. Wang, S. Chen, C. Zhang, Risk-based uncertainty set optimization method for energy management of hybrid ac/dc microgrids with uncertain renewable generation, IEEE Transactions on Smart Grid 11 (2) (2019) 1526-1542.

[13] L. Ji, G. Huang, Y. Xie, Y. Zhou, J. Zhou, Robust cost-risk tradeoff for day-ahead schedule optimization in residential microgrid system under worst-case conditional value-at-risk consideration, Energy 153 (2018) 324-337.

[14] X. Zheng, H. Chen, Y. Xu, Z. Liang, Y. Chen, A hierarchical method for robust scuc of multi-area power systems with novel uncertainty sets, IEEE Transactions on Power Systems 35 (2) (2019) 1364-1375.

[15] Y. Guan, J. Wang, Uncertainty sets for robust unit commitment, IEEE Transactions on Power Systems 
29 (3) (2013) 1439-1440.

[16] Y. Wang, Y. Yang, L. Tang, W. Sun, B. Li, A wasserstein based two-stage distributionally robust optimization model for optimal operation of cchp micro-grid under uncertainties, International Journal of Electrical Power \& Energy Systems 119 (2020) 105941.

[17] Y. Xiang, J. Liu, Y. Liu, Robust energy management of microgrid with uncertain renewable generation and load, IEEE Transactions on Smart Grid 7 (2) (2016) 1034-1043.

[18] T. Ding, J. Lv, R. Bo, Z. Bie, F. Li, Lift-and-project mvee based convex hull for robust sced with wind power integration using historical data-driven modeling approach, Renewable energy 92 (2016) 415-427.

[19] W. Wei, F. Liu, S. Mei, Y. Hou, Robust energy and reserve dispatch under variable renewable generation, IEEE Transactions on Smart Grid 6 (1) (2014) 369-380.

[20] Y. Zhang, X. Han, Y. Ming, M. Wang, Z. li, P. Ye, X. Bo, Distributionally robust unit commitment based on imprecise dirichlet model, Proceedings of the CSEE 39 (17) (2019) 5074-5084.

[21] M. Yang, J. Wang, H. Diao, J. Qi, X. Han, Interval estimation for conditional failure rates of transmission lines with limited samples, IEEE Transactions on Smart Grid 9 (4) (2016) 2752-2763.

[22] Y. Zhang, X. Han, M. Yang, B. Xu, Y. Zhao, H. Zhai, Adaptive robust unit commitment considering distributional uncertainty, International Journal of Electrical Power \& Energy Systems 104 (2019) 635-644.

[23] P. Walley, Statistical reasoning with imprecise probabilities. 1991 (1991).

[24] H. V. Haghi, S. Lotfifard, Spatiotemporal modeling of wind generation for optimal energy storage sizing, IEEE Transactions on Sustainable Energy 6 (1) (2014) 113-121.

[25] J. Cao, Z. Yan, Probabilistic optimal power flow considering dependences of wind speed among wind farms by pair-copula method, International Journal of Electrical Power \& Energy Systems 84 (2017) 296-307.

[26] Z. Xie, T. Ji, M. Li, Q. Wu, Quasi-monte carlo based probabilistic optimal power flow considering the correlation of wind speeds using copula function, IEEE Transactions on Power Systems 33 (2) (2017) 2239-2247.

[27] H. Chen, P. Xuan, Y. Wang, K. Tan, X. Jin, Key technologies for integration of multitype renewable energy sourcesresearch on multi-timeframe robust scheduling/dispatch, IEEE Transactions on Smart Grid 7 (1) (2015) 471-480.

[28] B. Zeng, L. Zhao, Solving two-stage robust optimization problems using a column-and-constraint generation method, Operations Research Letters 41 (5) (2013) 457-461.

[29] R. B. Nelsen, An introduction to copulas, Springer Science \& Business Media, 2007.

[30] M. S. Li, Z. J. Lin, T. Y. Ji, Q. H. Wu, Risk constrained stochastic economic dispatch considering dependence of multiple wind farms using pair-copula, Applied Energy 226 (2018) 967-978.

[31] Z. Lin, H. Chen, Q. Wu, W. Li, M. Li, T. Ji, Mean-tracking model based stochastic economic dispatch for power systems with high penetration of wind power, Energy (2019) 116826.

[32] P. Walley, Inferences from multinomial data: learning about a bag of marbles, Journal of the Royal Statistical Society: Series B (Methodological) 58 (1) (1996) 3-34.

[33] J.-M. Bernard, An introduction to the imprecise dirichlet model for multinomial data, International Journal of Approximate Reasoning 39 (2-3) (2005) 123-150.

[34] J. Tan, Q. Wu, Q. Hu, W. Wei, F. Liu, Adaptive robust energy and reserve co-optimization of integrated electricity and heating system considering wind uncertainty, Applied Energy 260 (2020) 114230.

[35] C. Zhao, Y. Guan, Data-driven stochastic unit commitment for integrating wind generation, IEEE Transactions on Power Systems 31 (4) (2015) 2587-2596.

[36] D. Bertsimas, E. Litvinov, X. A. Sun, J. Zhao, T. Zheng, Adaptive robust optimization for the security constrained unit commitment problem, IEEE transactions on power systems 28 (1) (2012) 52-63.

[37] M. Carrión, J. M. Arroyo, A computationally efficient mixed-integer linear formulation for the thermal unit commitment problem, IEEE Transactions on power systems 21 (3) (2006) 1371-1378.

[38] R. Jiang, J. Wang, Y. Guan, Robust unit commitment with wind power and pumped storage hydro, IEEE Transactions on Power Systems 27 (2) (2011) 800-810. 
[39] http: // motor. ece.iit.edu/ Data/ 118bus ro.xls.

[40] C. Genest, L.-P. Rivest, Statistical inference procedures for bivariate archimedean copulas, Journal of the American statistical Association 88 (423) (1993) 1034-1043.

[41] G. Díaz, A note on the multivariate archimedean dependence structure in small wind generation sites, Wind Energy 17 (8) (2014) 1287-1295. 